\title{
Structural, Electronic, and Vibrational Properties of Isoniazid and Its Derivative N-Cyclopentylidenepyridine-4-carbohydrazide: A Quantum Chemical Study
}

\author{
Anoop kumar Pandey, ${ }^{1}$ Abhishek Bajpai, ${ }^{2}$ Vikas Baboo, ${ }^{3}$ and Apoorva Dwivedi ${ }^{2}$ \\ ${ }^{1}$ Department of Physics, Government Danteshwari P. G. College, Dantewada, Chhattisgarh 494449, India \\ ${ }^{2}$ Department of Physics, Govt. Kakatiya P. G. College Jagdalpur, District Bastar, Chhattisgarh 494001, India \\ ${ }^{3}$ Department of Chemistry, Lucknow University, Lucknow, Uttar Pradesh 226007, India
}

Correspondence should be addressed to Apoorva Dwivedi; apoorvahri@gmail.com

Received 25 November 2013; Accepted 13 January 2014; Published 24 February 2014

Academic Editor: John R. Sabin

Copyright (C) 2014 Anoop kumar Pandey et al. This is an open access article distributed under the Creative Commons Attribution License, which permits unrestricted use, distribution, and reproduction in any medium, provided the original work is properly cited.

\begin{abstract}
Isoniazid (Laniazid, Nydrazid), also known as isonicotinylhydrazine (INH), is an organic compound that is the first-line medication in prevention and treatment of tuberculosis. The optimized geometry of the isoniazid and its derivative Ncyclopentylidenepyridine-4-carbohydrazide molecule has been determined by the method of density functional theory (DFT). For both geometry and total energy, it has been combined with B3LYP functionals having LANL2DZ and 6-311 G (d, p) as the basis sets. Using this optimized structure, we have calculated the infrared wavenumbers and compared them with the experimental data. The calculated wavenumbers by LANL2DZ are in an excellent agreement with the experimental values. On the basis of fully optimized ground-state structure, TDDFT//B3LYP/LANL2DZ calculations have been used to determine the low-lying excited states of isoniazid and its derivative. Based on these results, we have discussed the correlation between the vibrational modes and the crystalline structure of isoniazid and its derivative. A complete assignment is provided for the observed FTIR spectra. The molecular HOMO, LUMO composition, their respective energy gaps, and MESP contours/surfaces have also been drawn to explain the activity of isoniazid and its derivative.
\end{abstract}

\section{Introduction}

Tuberculosis remains a major worldwide health problem with at least 10 million new cases and 3 million deaths estimated to occur annually. Isoniazid (Laniazid, Nydrazid), also known as isonicotinylhydrazine (INH), is an organic compound that is the first-line medication in prevention and treatment of tuberculosis. The compound was first synthesized in the early 20th century [1] but its activity against tuberculosis was first reported in the early 1950s, and three pharmaceutical companies attempted unsuccessfully to simultaneously patent the drug [2]. With the introduction of isoniazid, a cure for tuberculosis was first considered reasonable. Isoniazid is available in tablet, syrup, and injectable forms (given intramuscularly or intravenously). It is available worldwide, is inexpensive, and is generally well tolerated. It is manufactured from isonicotinic acid, which is produced from 4-methylpyridine [3].

A previous investigation was done on determination of microquantities of isoniazid (INH), either in pure form or in pharmaceutical formulations by calorimetric method [4]. In another work, studies on the 4-benzoylpyridine-3carboxamide entity as a fragment model of the isoniazidNAD adduct were carried out [5]. A previous work on vibrational analysis of isoniazid was done by Gunasekaran et al. [6] by Wilson G.F. method which is a classical method, so it is necessary to work again with quantum chemical method to reduce the inaccuracy in previous work. The title compound, $\mathrm{N}$-cyclopentylidenepyridine-4-carbohydrazide, $\mathrm{C}_{11} \mathrm{H}_{13} \mathrm{~N}_{3} \mathrm{O}$, is a derivative of the antituberculosis drug isoniazid. The 
TABLE 1: Optimized geometrical parameters of isoniazid and N-cyclopentylidenepyridine-4-carbohydrazide (CPPC) by (B3LYP)/ 6-311 G (d, p) and (B3LYP)/LANL2DZ methods.

\begin{tabular}{|c|c|c|c|c|c|c|c|}
\hline \multicolumn{4}{|c|}{ Isoniazid } & \multicolumn{4}{|c|}{ N-Cyclopentylidenepyridine-4-carbohydrazide parameters } \\
\hline \multirow{3}{*}{ Parameters } & \multicolumn{6}{|c|}{ Bond lengths } & \multirow{3}{*}{ Exp. } \\
\hline & \multirow[b]{2}{*}{ LANL2DZ } & \multirow{2}{*}{$\begin{array}{l}\text { Calculated } \\
\text { 6-311 G (d, p) }\end{array}$} & \multirow[b]{2}{*}{$\begin{array}{l}\text { Wilson GF } \\
\text { (literature) }\end{array}$} & \multirow{2}{*}{ Parameters } & \multicolumn{2}{|c|}{ Calculated } & \\
\hline & & & & & LANL2DZ & $6-311 \mathrm{G}(\mathrm{d}, \mathrm{p})$ & \\
\hline$R(1,2)$ & 1.4036 & 1.3933 & $(1.470)$ & $R(1,2)$ & 1.4054 & 1.3905 & $(1.3801)$ \\
\hline$R(1,6)$ & 1.3599 & 1.3355 & $(1.358)$ & $R(1,6)$ & 1.3596 & 1.3381 & $(1.3309)$ \\
\hline$R(1,7)$ & 1.0866 & 1.0866 & $(1.100)$ & $R(1,7)$ & 1.0865 & 1.0862 & $(0.95)$ \\
\hline$R(2,3)$ & 1.4146 & 1.3965 & $(1.420)$ & $R(2,3)$ & 1.4100 & 1.3954 & (1.3836) \\
\hline$R(2,8)$ & 1.0845 & 1.0788 & $(1.100)$ & $R(2,8)$ & 1.0850 & 1.0824 & $(0.95)$ \\
\hline$R(3,4)$ & 1.4124 & 1.3972 & $(1.420)$ & $R(3,4)$ & 1.4119 & 1.397 & (1.3904) \\
\hline$R(3,11)$ & 1.5098 & 1.5067 & $(1.517)$ & $R(3,11)$ & 1.5114 & 1.5098 & (1.4973) \\
\hline$R(4,5)$ & 1.4088 & 1.3903 & $(1.420)$ & $R(4,5)$ & 1.4079 & 1.3937 & (1.3768) \\
\hline$R(4,9)$ & 1.0806 & 1.0822 & $(1.100)$ & $R(4,9)$ & 1.0868 & 1.084 & $(0.95)$ \\
\hline$R(5,6)$ & 1.3563 & 1.3373 & $(1.358)$ & $R(5,6)$ & 1.3564 & 1.3346 & $(1.3360)$ \\
\hline$R(5,10)$ & 1.0872 & 1.0864 & $(1.100)$ & $R(5,10)$ & 1.0870 & 1.0866 & $(0.95)$ \\
\hline$R(11,12)$ & 1.2625 & 1.2198 & (1.208) & $R(11,12)$ & 1.2496 & 1.2106 & (1.2249) \\
\hline$R(11,13)$ & 1.3903 & 1.377 & $(1.369)$ & $R(11,13)$ & 1.3920 & 1.3825 & (1.3484) \\
\hline$R(13,16)$ & 1.0203 & 1.0161 & $(1.012)$ & $R(13,14)$ & 1.3977 & 1.369 & (1.3961) \\
\hline$R(13,17)$ & 1.4052 & 1.402 & $(1.352)$ & $R(13,15)$ & 1.0175 & 1.0129 & $(0.889)$ \\
\hline$R(14,17)$ & 1.0170 & 1.0152 & $(-)$ & $R(14,16)$ & 1.2980 & 1.275 & (1.2759) \\
\hline$R(15,17)$ & 1.0170 & 1.0142 & $(-)$ & $R(16,17)$ & 1.5220 & 1.5146 & $(1.5076)$ \\
\hline- & - & - & - & $R(16,20)$ & 1.5329 & 1.5238 & (1.5035) \\
\hline- & - & - & - & $R(17,18)$ & 1.5495 & 1.5388 & (1.5243) \\
\hline- & - & - & - & $R(17,21)$ & 1.1002 & 1.0975 & $(0.99)$ \\
\hline- & - & - & - & $R(17,22)$ & 1.0942 & 1.0904 & $(0.99)$ \\
\hline - & - & - & - & $R(18,19)$ & 1.5529 & 1.5413 & $(1.5227)$ \\
\hline- & - & - & - & $R(18,23)$ & 1.0985 & 1.0952 & $(0.99)$ \\
\hline- & - & - & - & $R(18,24)$ & 1.0955 & 1.0915 & $(0.99)$ \\
\hline- & - & - & - & $R(19,20)$ & 1.5537 & 1.5438 & (1.5333) \\
\hline- & - & - & - & $R(19,25)$ & 1.0955 & 1.0915 & $(0.99)$ \\
\hline- & - & - & - & $R(19,26)$ & 1.0983 & 1.0946 & $(0.99)$ \\
\hline- & - & - & - & $R(20,27)$ & 1.1020 & 1.0983 & $(0.99)$ \\
\hline - & - & - & - & $R(20,28)$ & 1.1001 & 1.0955 & $(0.99)$ \\
\hline \multicolumn{8}{|c|}{ Bond angles } \\
\hline Parameters & LANL2DZ & 6-311 G $(d, p)$ & Wilson GF & Parameters & LANL2DZ & $6-311 \mathrm{G}(\mathrm{d}, \mathrm{p})$ & Exp. \\
\hline$A(2,1,6)$ & 123.156 & 124.085 & $(128.1)$ & $A(2,1,6)$ & 123.207 & 123.7612 & (124.11) \\
\hline$A(2,1,7)$ & 120.854 & 119.882 & $(115.9)$ & $A(2,1,7)$ & 120.864 & 120.2359 & $(117.9)$ \\
\hline$A(6,1,7)$ & 115.989 & 116.031 & $(115.9)$ & $A(6,1,7)$ & 115.927 & 116.0026 & $(117.9)$ \\
\hline$A(1,2,3)$ & 119.423 & 118.609 & (113.4) & $A(1,2,3)$ & 119.086 & 118.8914 & $(118.76)$ \\
\hline$A(1,2,8)$ & 121.333 & 121.238 & (123.3) & $A(1,2,8)$ & 121.357 & 121.5728 & (120.6) \\
\hline$A(3,2,8)$ & 119.243 & 120.148 & (123.3) & $A(3,2,8)$ & 119.553 & 119.5347 & $(120.6)$ \\
\hline$A(2,3,4)$ & 117.688 & 117.644 & $(120.0)$ & $A(2,3,4)$ & 118.003 & 117.7332 & $(118.04)$ \\
\hline$A(2,3,11)$ & 115.580 & 116.359 & (119.9) & $A(2,3,11)$ & 117.887 & 117.7499 & $(119.94)$ \\
\hline$A(4,3,11)$ & 126.731 & 125.851 & $(119.9)$ & $A(4,3,1)$ & 124.079 & 124.4757 & $(121.87)$ \\
\hline
\end{tabular}


TABle 1: Continued.

\begin{tabular}{|c|c|c|c|c|c|c|c|}
\hline$A(3,4,5)$ & 118.793 & 116.359 & (120.0) & $A(3,4,5)$ & 118.940 & 118.7973 & (118.51) \\
\hline$A(3,4,9)$ & 120.388 & 119.095 & $(119.90)$ & $A(3,4,9)$ & 122.012 & 121.7783 & (120.7) \\
\hline$A(5,4,9)$ & 120.818 & 119.585 & (119.9) & $A(5,4,9)$ & 119.000 & 119.3816 & $(120.7)$ \\
\hline$A(4,5,6)$ & 123.709 & 121.315 & (123.5) & $A(4,5,6)$ & 123.267 & 123.7549 & $(124.23)$ \\
\hline$A(4,5,10)$ & 120.395 & 120.222 & (118.2) & $A(4,5,1)$ & 120.733 & 120.095 & $(117.9)$ \\
\hline$A(6,5,10)$ & 115.895 & 116.119 & (118.2) & $A(6,5,1)$ & 115.997 & 116.1492 & $(117.9)$ \\
\hline$A(1,6,5)$ & 117.229 & 116.890 & (115.9) & $A(1,6,5)$ & 117.480 & 117.0429 & $(116.34)$ \\
\hline$A(3,11,12)$ & 120.109 & 120.608 & (123.5) & $A(3,11,12)$ & 121.407 & 121.7922 & (121.01) \\
\hline$A(3,11,13)$ & 123.160 & 120.465 & (113.2) & $A(3,11,13)$ & 114.386 & 113.8995 & (115.24) \\
\hline$A(12,11,13)$ & 116.730 & 116.119 & $(123.10)$ & $A(12,11,13)$ & 124.194 & 124.3057 & (123.71) \\
\hline$A(11,13,16)$ & 112.897 & 110.815 & (118.3) & $A(11,13,14)$ & 120.222 & 120.4885 & (117.90) \\
\hline$A(11,13,17)$ & 127.415 & 123.657 & (120.9) & $A(11,13,15)$ & 119.204 & 118.6787 & (120.9) \\
\hline$A(16,13,17)$ & 119.687 & 120.608 & (120.9) & $A(14,13,15)$ & 120.413 & 120.0841 & $(121.2)$ \\
\hline$A(13,17,14)$ & 113.970 & 112.315 & $(-)$ & $A(13,14,16)$ & 117.618 & 117.4204 & (116.39) \\
\hline$A(13,17,15)$ & 113.972 & 109.553 & $(-)$ & $A(14,16,17)$ & 120.864 & 121.3315 & (120.59) \\
\hline$A(14,17,15)$ & 115.002 & 109.146 & $(-)$ & $A(14,16,20)$ & 129.064 & 128.9664 & $(129.82)$ \\
\hline- & - & - & - & $A(17,16,20)$ & 110.061 & 109.6831 & (109.59) \\
\hline - & - & - & - & $A(16,17,18)$ & 103.828 & 103.7083 & $(103.49)$ \\
\hline- & - & - & - & $A(16,17,21)$ & 109.558 & 109.1963 & $(111.1)$ \\
\hline- & - & - & - & $A(16,17,22)$ & 111.559 & 111.673 & $(111.1)$ \\
\hline- & - & - & - & $A(18,17,21)$ & 110.424 & 110.1309 & $(111.1)$ \\
\hline- & - & - & - & $A(18,17,22)$ & 114.254 & 114.7737 & (111.1) \\
\hline- & - & - & - & $A(21,17,22)$ & 107.188 & 107.2821 & (109) \\
\hline - & - & - & - & $A(17,18,19)$ & 103.849 & 103.6438 & (103.09) \\
\hline - & - & - & - & $A(17,18,23)$ & 110.096 & 109.8783 & $(111.1)$ \\
\hline- & - & - & - & $A(17,18,24)$ & 112.690 & 113.1144 & (111.1) \\
\hline- & - & - & - & $A(19,18,23)$ & 110.044 & 109.8696 & $(111.1)$ \\
\hline- & - & - & - & $A(19,18,24)$ & 112.485 & 112.7897 & $(111.1)$ \\
\hline- & - & - & - & $A(23,18,24)$ & 107.663 & 107.5175 & $(109.1)$ \\
\hline - & - & - & - & $A(18,19,20)$ & 104.367 & 104.3214 & $(103.63)$ \\
\hline - & - & - & - & $A(18,19,25)$ & 112.900 & 113.1836 & (111) \\
\hline- & - & - & - & $A(18,19,26)$ & 109.818 & 109.665 & (111) \\
\hline - & - & - & - & $A(20,19,25)$ & 112.372 & 112.7006 & (111) \\
\hline- & - & - & - & $A(20,19,26)$ & 109.763 & 109.5735 & (111) \\
\hline- & - & - & - & $A(25,19,26)$ & 107.597 & 107.3627 & (109) \\
\hline- & - & - & - & $A(16,20,19)$ & 104.150 & 104.3483 & (103.78) \\
\hline- & - & - & - & $A(16,20,27)$ & 109.9266 & 109.6136 & (111) \\
\hline- & - & - & - & $A(16,20,28)$ & 112.6795 & 112.4489 & (111) \\
\hline - & - & - & - & $A(19,20,27)$ & 110.6596 & 110.8512 & (111) \\
\hline - & - & - & - & $A(19,20,28)$ & 112.2491 & 112.6471 & (111) \\
\hline- & - & - & - & $A(27,20,28)$ & 107.209 & 106.9667 & $(109)$ \\
\hline
\end{tabular}

XRD analysis of this compound was done recently by Lemmerer et al. [7]. It is a derivative of such an important biological isoniazid molecule so comparative study of these two compounds is necessary for discovery of new drugs.

In the present communication, the molecular structure of well-known drug isoniazid and its derivative has been analyzed using the density functional theory. The equilibrium geometry, harmonic vibrational frequencies, and HOMOLUMO gap have been calculated by the density functional B3LYP method employing LANL2DZ and 6-311 G (d, p) as the basis sets. The detailed interpretation of the infrared spectra of isoniazid and its derivative in terms of the normal mode analysis has been reported. The main objective of the present study is to investigate in detail the vibrational 
TABLE 2: Comparison of the observed and calculated vibrational spectra of isoniazid with (B3LYP)/6-311 G (d, p) and B3LYP/LANL2DZ.

\begin{tabular}{|c|c|c|c|c|c|c|}
\hline \multicolumn{4}{|c|}{ B3LYP } & \multirow{2}{*}{ FTIR (exp.) } & \multirow{2}{*}{ FT Raman (exp.) } & \multirow{2}{*}{ Vibrational assignments } \\
\hline LANL2DZ & IR (Int.) & 6-311 G (d, p) & IR (Int.) & & & \\
\hline 27 & 4.4893 & 39 & 2.1136 & - & - & Whole molecule twists from joint \\
\hline 136 & 0.1522 & 121 & 3.0144 & - & - & Twist NH2 \\
\hline 163 & 0.0223 & 134 & 2.9603 & - & - & Rock NH2 \\
\hline 200 & 2.6928 & 198 & 2.2873 & - & - & Whole molecule bends from joint \\
\hline 281 & 9.3909 & 261 & 15.6415 & - & - & $\beta(\mathrm{C}-\mathrm{N}-\mathrm{N})$ \\
\hline 343 & 64.239 & 306 & 54.4552 & - & - & Twist NH2 \\
\hline 380 & 4.8231 & 370 & 2.1727 & - & - & $\tau(\mathrm{C}-\mathrm{C}-\mathrm{C}=\mathrm{O})$ \\
\hline 385 & 3.0767 & 377 & 0.0835 & - & - & $\gamma(\mathrm{C}-\mathrm{C}-\mathrm{C})+\gamma(\mathrm{C}-\mathrm{C}-\mathrm{H})$ \\
\hline 457 & 3.736 & 452 & 5.6374 & 504 & 504 & $\gamma(\mathrm{C}-\mathrm{C}-\mathrm{C})+\gamma(\mathrm{C}-\mathrm{C}-\mathrm{H})$ \\
\hline 528 & 2.5885 & 544 & 17.1188 & - & - & Rock NH2 $+\beta(\mathrm{C}-\mathrm{C}=\mathrm{O})$ \\
\hline 578 & 142.03 & 588 & 52.8091 & - & - & Rock NH2 \\
\hline 658 & 23.445 & 636 & 26.3687 & 660 & 666 & $\tau(\mathrm{C}-\mathrm{C}-\mathrm{C}-\mathrm{C})+\tau(\mathrm{C}-\mathrm{N}-\mathrm{C}-\mathrm{C})$ \\
\hline 659 & 28.530 & 659 & 0.79 & & & Twist NH2 \\
\hline 668 & 162.33 & 702 & 45.9836 & 674 & 682 & Rock NH2 \\
\hline 701 & 110.45 & 720 & 3.2826 & - & - & $\gamma(\mathrm{C}-\mathrm{N}-\mathrm{H})$ \\
\hline 710 & 0.1352 & 745 & 774.0493 & - & - & $\beta(\mathrm{C}-\mathrm{N}-\mathrm{C})+\beta(\mathrm{C}-\mathrm{C}-\mathrm{C})$ \\
\hline 758 & 29.601 & 836 & 13.6245 & 745 & 750 & $\gamma(\mathrm{C}-\mathrm{C}-\mathrm{C})+\gamma(\mathrm{C}-\mathrm{C}-\mathrm{H})$ \\
\hline 854 & 37.843 & 865 & 0.5422 & 845 & 849 & $\gamma(\mathrm{C}-\mathrm{C}-\mathrm{H})$ \\
\hline 904 & 0.0237 & 874 & 143.4664 & - & - & $\gamma(\mathrm{C}-\mathrm{C}-\mathrm{H})$ \\
\hline 953 & 4.925 & 956 & 0.4745 & - & - & Ring breathing \\
\hline 998 & 2.1689 & 970 & 3.0528 & - & - & $\gamma(\mathrm{C}-\mathrm{C}-\mathrm{H})$ \\
\hline 1005 & 2.381 & 977 & 3.1906 & 1020 & 1002 & $\gamma(\mathrm{C}-\mathrm{C}-\mathrm{H})$ \\
\hline 1046 & 9.4117 & 1052 & 17.7683 & & & $\beta(\mathrm{C}-\mathrm{C}-\mathrm{H})+\beta(\mathrm{C}-\mathrm{C}=\mathrm{O})$ \\
\hline 1049 & 20.656 & 1060 & 5.0938 & 1061 & 1057 & $\beta(\mathrm{C}-\mathrm{N}-\mathrm{C})+\beta(\mathrm{C}-\mathrm{C}-\mathrm{C})$ \\
\hline 1072 & 1.5593 & 1071 & 3.1435 & - & - & $\beta(\mathrm{C}-\mathrm{C}-\mathrm{H})$ \\
\hline 1170 & 69.756 & 1154 & 50.7026 & - & - & $\beta(\mathrm{C}-\mathrm{C}-\mathrm{C})+\beta(\mathrm{C}-\mathrm{C}-\mathrm{H})$ \\
\hline 1200 & 1.2477 & 1198 & 1.8033 & 1200 & 1187 & $\beta(\mathrm{C}-\mathrm{C}-\mathrm{H})$ \\
\hline 1223 & 0.006 & 1227 & 3.1093 & - & - & Twist NH2 \\
\hline 1249 & 5.3368 & 1268 & 3.7348 & - & - & $\nu(\mathrm{C}-\mathrm{N})+\nu(\mathrm{C}-\mathrm{C})$ \\
\hline 1305 & 3.9465 & 1301 & 3.2532 & - & - & $\beta(\mathrm{C}-\mathrm{C}-\mathrm{H})$ \\
\hline 1353 & 177.39 & 1334 & 194.7891 & 1334 & 1331 & $\beta(\mathrm{O}=\mathrm{C}-\mathrm{N})+\beta(\mathrm{C}-\mathrm{C}-\mathrm{H})$ \\
\hline 1383 & 56.249 & 1386 & 31.6275 & - & - & $\beta(\mathrm{C}-\mathrm{C}-\mathrm{H})$ \\
\hline 1416 & 48.473 & 1427 & 19.6455 & 1412 & 1410 & $\beta(\mathrm{C}-\mathrm{N}-\mathrm{H})$ \\
\hline 1448 & 2.1058 & 1465 & 1.3185 & - & - & $\beta(\mathrm{C}-\mathrm{C}-\mathrm{H})$ \\
\hline 1517 & 56.958 & 1535 & 28.9279 & 1472 & - & $\nu(\mathrm{C}-\mathrm{C})$ \\
\hline 1560 & 11.455 & 1574 & 9.7845 & - & - & $\nu(\mathrm{C}-\mathrm{C})$ \\
\hline 1577 & 87.192 & 1634 & 30.7552 & 1556 & 1551 & $\nu(\mathrm{C}=\mathrm{O})$ \\
\hline 1642 & 55.629 & 1684 & 235.7803 & 1635 & 1642 & $\mathrm{~S}(\mathrm{NH} 2)$ \\
\hline 3079 & 32.086 & 3030 & 34.8746 & 3050 & 3065 & $v(\mathrm{C}-\mathrm{H})$ \\
\hline 3085 & 9.0201 & 3035 & 14.6458 & - & - & $v(\mathrm{C}-\mathrm{H})$ \\
\hline 3121 & 4.9876 & 3089 & 2.9337 & - & - & $v(\mathrm{C}-\mathrm{H})$ \\
\hline 3153 & 8.4279 & 3119 & 3.3617 & - & - & $v(\mathrm{C}-\mathrm{H})$ \\
\hline 3381 & 1.8185 & 3341 & 1.8712 & - & - & $\nu(\mathrm{N}-\mathrm{H})$ \\
\hline 3389 & 17.234 & 3387 & 16.6022 & 3303 & 3300 & $\nu(\mathrm{N}-\mathrm{H})$ \\
\hline 3509 & 9.0098 & 3426 & 4.449 & - & - & $\nu(\mathrm{N}-\mathrm{H})$ \\
\hline
\end{tabular}

$\nu$ : stretching; $\beta$ : in plane bending; $\gamma$ : out of plane bending; S: scissoring; w: wagging; $\tau$ : torsion. 
TABLE 3: Comparison of calculated vibrational spectra of N-cyclopentylidenepyridine-4-carbohydrazide (CPPC) with (B3LYP)/6-311 G (d, p) and B3LYP/LANL2DZ.

\begin{tabular}{|c|c|c|c|c|}
\hline \multirow[b]{2}{*}{ LANL2DZ } & \multicolumn{2}{|c|}{ B3LYP } & \multirow[b]{2}{*}{ IR (Int.) } & \multirow{2}{*}{ Vibrational assignments } \\
\hline & IR (Int.) & 6-311 G (d, p) & & \\
\hline 29 & 0.189 & 29 & 0.0368 & Whole molecule twists from middle \\
\hline 44 & 0.0329 & 43 & 0.0041 & Whole molecule twists from middle \\
\hline 55 & 1.0643 & 56 & 0.5335 & Ring B bends from middle \\
\hline 65 & 2.6343 & 64 & 1.7946 & Twisting in ring B \\
\hline 115 & 1.2256 & 113 & 0.2943 & Twisting in ring B \\
\hline 155 & 9.5443 & 152 & 8.6203 & Both rings twist from middle \\
\hline 189 & 11.479 & 187 & 10.7292 & Bending in whole molecule \\
\hline 221 & 0.5418 & 226 & 0.7907 & $\gamma(\mathrm{C}-\mathrm{C}-\mathrm{C})$ ring $\mathrm{B}$ \\
\hline 239 & 1.4716 & 243 & 1.22 & $\tau(\mathrm{C}-\mathrm{C}-\mathrm{N}-\mathrm{N})$ \\
\hline 284 & 4.2682 & 285 & 4.0485 & $\tau(\mathrm{N}-\mathrm{N}-\mathrm{C}=\mathrm{O})$ \\
\hline 324 & 4.6942 & 325 & 0.6016 & Twist $\mathrm{CH}_{2}$ ring $\mathrm{B}$ \\
\hline 376 & 0.124 & 374 & 0.0949 & Out of plane bending in ring $\mathrm{A}$ as a whole \\
\hline 395 & 2.2666 & 382 & 7.9387 & $\tau(\mathrm{C}-\mathrm{C}-\mathrm{C}=\mathrm{O})$ \\
\hline 453 & 8.0728 & 443 & 26.6387 & $\omega(\mathrm{N}-\mathrm{H})$ \\
\hline 466 & 11.181 & 464 & 11.8231 & Out of plane bending in whole molecule \\
\hline 542 & 4.2055 & 492 & 20.6542 & Whole molecule bends from middle \\
\hline 554 & 52.148 & 546 & 8.846 & Twist $\mathrm{CH}_{2}$ ring $\mathrm{B}$ \\
\hline 573 & 25.912 & 559 & 0.4785 & Twist $\mathrm{CH}_{2}$ ring $\mathrm{B}$ \\
\hline 588 & 7.2812 & 593 & 9.2922 & $\beta(\mathrm{C}-\mathrm{C}=\mathrm{N})$ \\
\hline 654 & 1.136 & 657 & 0.8099 & Ring A deformation \\
\hline 660 & 43.182 & 663 & 50.3605 & Ring A breathing \\
\hline 690 & 28.825 & 696 & 16.6761 & $\gamma(\mathrm{C}-\mathrm{C}-\mathrm{C})$ ring $\mathrm{A}$ \\
\hline 739 & 0.3987 & 736 & 0.1654 & Twist in ring $\mathrm{B}$ \\
\hline 750 & 13.234 & 746 & 10.518 & $\gamma(\mathrm{C}-\mathrm{C}-\mathrm{C})$ ring $\mathrm{A}$ \\
\hline 811 & 0.675 & 806 & 0.01 & $\gamma\left(\mathrm{CH}_{2}\right)$ ring $\mathrm{B}$ \\
\hline 835 & 12.940 & 825 & 13.797 & $\gamma(\mathrm{C}-\mathrm{C}-\mathrm{C}) \mathrm{rRing} \mathrm{B}$ \\
\hline 837 & 25.375 & 830 & 1.1714 & $\gamma(\mathrm{C}-\mathrm{C}-\mathrm{C})$ ring $\mathrm{A}$ \\
\hline 873 & 2.6331 & 866 & 2.3196 & $\gamma(\mathrm{C}-\mathrm{C}-\mathrm{C}) \operatorname{ring} \mathrm{B}$ \\
\hline 885 & 25.947 & 867 & 0.4876 & $\beta(\mathrm{N}-\mathrm{C}=\mathrm{O})$ joint to both rings \\
\hline 887 & 3.846 & 889 & 4.1429 & $\gamma(\mathrm{C}-\mathrm{C}-\mathrm{H})$ ring $\mathrm{A}$ \\
\hline 906 & 5.1907 & 907 & 22.5923 & Twist $\mathrm{CH}_{2}$ ring $\mathrm{B}$ \\
\hline 947 & 5.0577 & 933 & 2.7875 & $\gamma(\mathrm{C}-\mathrm{C}-\mathrm{H})$ ring $\mathrm{B}$ \\
\hline 951 & 5.9695 & 951 & 0.2745 & Ring A breathing \\
\hline 966 & 2.0181 & 973 & 4.7925 & $\gamma(\mathrm{C}-\mathrm{C}-\mathrm{C})$ ring $\mathrm{A}$ \\
\hline 986 & 18.651 & 974 & 0.9688 & $\gamma(\mathrm{C}-\mathrm{C}-\mathrm{C})$ ring $\mathrm{A}$ \\
\hline 990 & 12.259 & 985 & 0.3854 & $\gamma(\mathrm{C}-\mathrm{C}-\mathrm{C})$ ring $\mathrm{A}$ \\
\hline 1002 & 2.3552 & 995 & 0.0806 & Twist $\mathrm{CH}_{2}$ ring $\mathrm{B}$ \\
\hline 1027 & 5.8621 & 1021 & 12.9074 & $\gamma(\mathrm{C}-\mathrm{C}-\mathrm{C}) \operatorname{ring} \mathrm{B}$ \\
\hline 1048 & 14.225 & 1050 & 17.0033 & $\beta(\mathrm{C}-\mathrm{C}-\mathrm{H})$ ring $\mathrm{A}$ \\
\hline 1072 & 17.410 & 1067 & 8.9467 & $\beta(\mathrm{C}-\mathrm{C}-\mathrm{H})$ ring $\mathrm{A}$ \\
\hline 1104 & 73.216 & 1105 & 114.2174 & $\beta(C-C-H)$ ring $A$ \\
\hline 1127 & 0.6851 & 1118 & 0.8245 & Twist $\mathrm{CH}_{2}$ ring $\mathrm{B}$ \\
\hline 1144 & 9.5501 & 1144 & 23.861 & $\gamma(\mathrm{C}-\mathrm{C}-\mathrm{H})$ ring $\mathrm{B}$ \\
\hline 1184 & 15.684 & 1174 & 13.5043 & $\beta(C-C=N)$ ring $A+\beta(C-C-C)$ ring $B$ \\
\hline 1196 & 6.0684 & 1188 & 9.5816 & $\gamma(\mathrm{C}-\mathrm{C}-\mathrm{H})$ ring $\mathrm{B}$ \\
\hline
\end{tabular}


TABLE 3: Continued.

\begin{tabular}{|c|c|c|c|c|}
\hline \multirow[b]{2}{*}{ LANL2DZ } & \multicolumn{2}{|c|}{ B3LYP } & \multirow[b]{2}{*}{ IR (Int.) } & \multirow{2}{*}{ Vibrational assignments } \\
\hline & IR (Int.) & $6-311 \mathrm{G}(\mathrm{d}, \mathrm{p})$ & & \\
\hline 1203 & 3.3536 & 1195 & 23.0436 & $\beta(\mathrm{C}-\mathrm{C}-\mathrm{H})$ ring $\mathrm{A}$ \\
\hline 1222 & 62.461 & 1209 & 146.2051 & Twist $\mathrm{CH}_{2}$ ring $\mathrm{B}$ \\
\hline 1237 & 163.03 & 1217 & 51.1901 & $\beta(\mathrm{C}-\mathrm{C}-\mathrm{C})$ ring $\mathrm{A}+$ twist $\mathrm{CH}_{2}$ ring $\mathrm{B}$ \\
\hline 1249 & 10.424 & 1232 & 34.5492 & Ring A deformation \\
\hline 1266 & 9.0924 & 1256 & 4.27 & Twist $\mathrm{CH}_{2}$ ring $\mathrm{B}$ \\
\hline 1283 & 2.845 & 1279 & 0.3718 & Rock $\mathrm{CH}_{2}$ ring $\mathrm{B}$ \\
\hline 1305 & 0.2089 & 1295 & 0.4946 & Twist $\mathrm{CH}_{2}$ ring $\mathrm{B}$ \\
\hline 1306 & 0.0683 & 1297 & 1.5912 & Twist $\mathrm{CH}_{2}$ ring $\mathrm{B}$ \\
\hline 1312 & 0.6944 & 1301 & 0.9643 & $\beta(\mathrm{C}-\mathrm{C}-\mathrm{H})$ ring $\mathrm{A}$ \\
\hline 1381 & 13.894 & 1385 & 14.4629 & $\beta(C-C-H)$ ring $A$ \\
\hline 1436 & 34.372 & 1416 & 17.0927 & $\mathrm{~S}\left(\mathrm{CH}_{2}\right)$ ring $\mathrm{B}$ \\
\hline 1441 & 13.808 & 1420 & 7.2964 & $\mathrm{~S}\left(\mathrm{CH}_{2}\right)$ ring $\mathrm{B}$ \\
\hline 1447 & 11.264 & 1442 & 5.421 & $\beta(C-C-H)$ ring $A$ \\
\hline 1461 & 9.7004 & 1456 & 3.4055 & $\mathrm{~S}\left(\mathrm{CH}_{2}\right)$ ring $\mathrm{B}$ \\
\hline 1469 & 1.2504 & 1463 & 17.0394 & $\mathrm{~S}\left(\mathrm{CH}_{2}\right)$ ring $\mathrm{B}$ \\
\hline 1487 & 313.60 & 1489 & 318.044 & $\beta(\mathrm{C}-\mathrm{N}-\mathrm{H})$ \\
\hline 1529 & 37.617 & 1538 & 23.3689 & Ring A deformation \\
\hline 1563 & 7.4055 & 1573 & 17.1708 & $v(\mathrm{C}-\mathrm{C})$ ring $\mathrm{A}$ \\
\hline 1604 & 227.40 & 1654 & 22.9328 & $\nu(\mathrm{C}=\mathrm{O})$ \\
\hline 1623 & 2.596 & 1715 & 302.3136 & $v(\mathrm{C}=\mathrm{N})$ joint ring $\mathrm{B}$ \\
\hline 2902 & 18.706 & 2890 & 12.407 & $\nu(\mathrm{C}-\mathrm{H})$ ring $\mathrm{B}$ \\
\hline 2925 & 15.584 & 2900 & 12.3498 & $v(\mathrm{C}-\mathrm{H})$ ring $\mathrm{B}$ \\
\hline 2938 & 21.950 & 2917 & 25.4822 & $v(\mathrm{C}-\mathrm{H})$ ring $\mathrm{B}$ \\
\hline 2939 & 41.648 & 2923 & 12.8395 & $\nu(\mathrm{C}-\mathrm{H})$ ring $\mathrm{B}$ \\
\hline 2954 & 38.945 & 2934 & 41.6752 & $v(\mathrm{C}-\mathrm{H})$ ring $\mathrm{B}$ \\
\hline 3001 & 42.309 & 2975 & 40.8297 & $v(\mathrm{C}-\mathrm{H})$ ring $\mathrm{B}$ \\
\hline 3009 & 30.010 & 2981 & 39.1011 & $v(\mathrm{C}-\mathrm{H})$ ring $\mathrm{B}$ \\
\hline 3017 & 50.383 & 2990 & 14.2807 & $v(\mathrm{C}-\mathrm{H})$ ring $\mathrm{B}$ \\
\hline 3072 & 8.0634 & 3031 & 21.1113 & $\nu(\mathrm{C}-\mathrm{H})$ ring $\mathrm{A}$ \\
\hline 3086 & 26.263 & 3038 & 23.6222 & $v(\mathrm{C}-\mathrm{H})$ ring $\mathrm{A}$ \\
\hline 3095 & 16.702 & 3061 & 12.3221 & $\nu(\mathrm{C}-\mathrm{H})$ ring $\mathrm{A}$ \\
\hline 3118 & 4.5408 & 3088 & 3.1852 & $v(\mathrm{C}-\mathrm{H})$ ring $\mathrm{A}$ \\
\hline 3416 & 17.207 & 3412 & 13.2313 & $\nu(\mathrm{N}-\mathrm{H})$ \\
\hline
\end{tabular}

$\nu$ : stretching; $\beta$ : in plane bending; $\gamma$ : out of plane bending; S: scissoring; $\omega$ : wagging; $\tau$ : torsion.

spectra of important biological molecules (isoniazid and its derivative) by DFT [8] method, which can presumably help in understanding its dynamical behavior. To the best of our knowledge no detailed DFT calculations have been performed on isoniazid and its derivative so far in the literature. Here we have discussed only results from LANL2DZ because these results are closer to experimental data than 6-311 G (d, p). So, here LANL2DZ shows supremacy over 6-311 G (d, p) basis set.

\section{Experimental and Computational Methods}

2.1. Structure and Spectra. The molecular structures of the title compounds isoniazid and its derivative are made by molecular modeling. The model molecular structures of the compounds are given in Figures 1 and 2. Fourier transform infrared spectrum was recorded with FTIR Perkin Elmer spectrometer in $\mathrm{KBr}$ dispersion in the range of 400 to $4000 \mathrm{~cm}^{-1}$ for the title molecule. Fourier transform Raman spectrum was recorded in the range of 50 to $3500 \mathrm{~cm}^{-1}$ for isoniazid [6].

2.2. Computational Methods. Initial geometry was generated from standard geometrical parameters and was minimized without any constraint in the potential energy surface. The gradient corrected density functional theory (DFT) with the three-parameter hybrid functional (B3) [9] for the exchange part and the Lee-Yang-Parr (LYP) correlation function [10] has been employed for the computation of molecular structure, vibrational frequencies, HOMO-LUMO, and energies of the optimized structures, using GAUSSIAN 09 [11]. The calculated vibrational frequencies have also been scaled 
TABLE 4: Calculated parameters for isoniazid and CPPC using TDDFT//B3LYP/LANL2DZ.

\begin{tabular}{|c|c|c|c|c|}
\hline Excitation & CI coefficient & $\begin{array}{c}\text { Expansion wave length }(\mathrm{nm}) \\
\text { calculated }\end{array}$ & Oscillator strength & Energy $(\mathrm{eV})$ \\
\hline \multicolumn{5}{|l|}{ Isoniazid } \\
\hline \multicolumn{5}{|l|}{ Excited state 1} \\
\hline $31 \rightarrow 37$ & 0.63973 & 214.82 & 0.1780 & 5.7714 \\
\hline $34 \rightarrow 38$ & 0.27134 & & & \\
\hline \multicolumn{5}{|l|}{ Excited state 2} \\
\hline $34 \rightarrow 38$ & 0.50322 & 180.83 & 0.5155 & 6.8565 \\
\hline $31 \rightarrow 38$ & -0.13282 & & & \\
\hline \multicolumn{5}{|l|}{ Excited state 3} \\
\hline $34 \rightarrow 39$ & 0.53619 & 159.44 & 0.2444 & 7.7762 \\
\hline $29 \rightarrow 38$ & -0.11809 & & & \\
\hline \multicolumn{5}{|l|}{ СРPC } \\
\hline \multicolumn{5}{|l|}{ Excited state 1} \\
\hline $49 \rightarrow 55$ & 0.45328 & 214.82 & 0.3239 & 5.7716 \\
\hline $53 \rightarrow 57$ & 0.10859 & & & \\
\hline $52 \rightarrow 57$ & 0.10042 & & & \\
\hline \multicolumn{5}{|c|}{ Excited state 2} \\
\hline $47 \rightarrow 55$ & 0.26652 & 185.18 & 0.2676 & 6.6955 \\
\hline $50 \rightarrow 56$ & 0.50556 & & & \\
\hline $53 \rightarrow 58$ & 0.22945 & & & \\
\hline \multicolumn{5}{|l|}{ Excited state 3} \\
\hline $49 \rightarrow 56$ & 0.45774 & 175.46 & 0.1706 & 7.0662 \\
\hline $50 \rightarrow 57$ & 0.41085 & & & \\
\hline
\end{tabular}

TABLE 5: Lowest energy, HOMO-LUMO gap (frontier orbital energy gap) and dipole moment of isoniazid and N-cyclopentylidenepyridine4-carbohydrazide (CPPC) by (B3LYP)/6-311 G (d, p) and (B3LYP)/LANL2DZ methods.

\begin{tabular}{lcccc}
\hline \multirow{2}{*}{ Parameters } & \multicolumn{2}{c}{ Isoniazid } & \multicolumn{2}{c}{ N-Cyclopentylidenepyridine-4-carbohydrazide } \\
& LANL2DZ & $6-311 \mathrm{G}(\mathrm{d}, \mathrm{p})$ & LANL2DZ & $6-311 \mathrm{G}(\mathrm{d}, \mathrm{p})$ \\
\hline Energy (in au) & -472.2226 & -472.4245 & -666.3336 & -666.6202 \\
Dipole moment (in Debye) & 5.49 & 4.94 & 6.25 & 5.37 \\
HOMO & -6.8353 & -6.8852 & -6.7673 & -6.7821 \\
LUMO & -1.8800 & -1.9129 & -1.9815 & -1.9875 \\
Frontier orbital energy gap (eV) & 4.9552 & 4.9723 & 4.7857 & 4.7946 \\
\hline
\end{tabular}

by a factor of 0.963 [12]. By combining the results of GaussView's program [13] with symmetry considerations, vibrational frequency assignments were made with a high degree of accuracy. We used this approach for the prediction of IR frequencies of title compound and found it to be very straightforward. Density functional theory calculations are reported to provide excellent vibrational frequencies of organic compound if the calculated frequencies are scaled to compensate for the approximate treatment of electron correlation, for basis set deficiencies and for anharmonicity. A number of studies have been carried out regarding calculations of vibrational spectra by using B3LYP methods with LANL2DZ and 6-311 G (d, p) basis sets. The scaling factor was applied successfully for B3LYP method and was found to be easily transferable in a number of molecules. Thus, vibrational frequencies calculated by using the B3LYP functional with LANL2DZ as basis set can be utilized to eliminate the uncertainties in the fundamental assignment in the IR spectra.

\section{Results and Discussion}

3.1. Molecular Structure. In the case of isoniazid, the molecule has only one pyridine ring. The optimized bond length of $\mathrm{C}-\mathrm{C}$ in six membered pyridine ring ranges between $1.403 \AA$ and $1.414 \AA$. The optimized bond length of $\mathrm{C}-\mathrm{C}$ outside the ring is $1.509 \AA$. The optimized bond lengths of $\mathrm{C}-$ $\mathrm{N}$ in ring range between $1.356 \AA$ and $1.359 \AA$. The optimized bond length of $\mathrm{C}-\mathrm{N}$ outside the ring is found to be $1.390 \AA$. The optimized $\mathrm{C}-\mathrm{H}$ bond lengths attached to the ring are found to be $1.080 \AA-1.087 \AA$. The optimized $\mathrm{C}=\mathrm{O}$ bond length adjacent to ring is found to be $1.262 \AA$. The optimized $\mathrm{N}-\mathrm{H}$ bond lengths adjacent to ring are found to be $1.016 \AA$ $1.020 \AA$. 
TABLE 6: Calculated $\varepsilon_{\text {HOMO }}, \varepsilon_{\text {LUMO }}$, energy band gap $\left(\varepsilon_{\mathrm{L}}-\varepsilon_{\mathrm{H}}\right)$, chemical potential $(\mu)$, electronegativity $(\chi)$, global hardness $(\eta)$, global softness $(S)$, and global electrophilicity index $(\omega)$ for isoniazid and CPPC at B3LYP/LANL2DZ level.

\begin{tabular}{|c|c|c|c|c|c|c|c|c|}
\hline Molecules & $\varepsilon_{\mathrm{H}}$ & $\varepsilon_{\mathrm{L}}$ & $\varepsilon_{\mathrm{L}}-\varepsilon_{\mathrm{H}}$ & $\chi$ & $\mu$ & $\eta$ & $S$ & $\omega$ \\
\hline CPPC 2 & -6.7673 & -1.9815 & 4.7857 & 4.3744 & -4.3744 & 2.3929 & 0.2090 & 3.9984 \\
\hline ISO 2 & -6.8353 & -1.8800 & 4.9552 & 4.3577 & -4.3577 & 2.4776 & 0.2018 & 3.8322 \\
\hline
\end{tabular}

TABLE 7: Fukui functions $\left(f_{k}^{+}, f_{k}^{-}\right)$, local softness $\left(s_{k}{ }^{+}, s_{k}{ }^{-}\right)$, and local electrophilicity indices $\left(\omega_{k}{ }^{+}, \omega_{k}{ }^{-}\right)$for selected atomic sites of CPPC, using Mulliken population analysis at B3LYP/LANL2DZ level.

\begin{tabular}{|c|c|c|c|c|c|c|}
\hline CPPC & $f_{k}^{+}$ & $f_{k}^{-}$ & $s_{k}^{+}$ & $s_{k}^{-}$ & $\omega_{k}^{+}$ & $\omega_{k}^{-}$ \\
\hline N6 & 0.063725 & 0.047259 & 0.013319 & 0.009877 & 0.254798 & 0.18896 \\
\hline C11 & 0.088784 & 0.050459 & 0.018556 & 0.010546 & 0.354994 & 0.201755 \\
\hline N13 & 0.016223 & 0.049147 & 0.003391 & 0.010272 & 0.064866 & 0.196509 \\
\hline N14 & 0.023381 & 0.043409 & 0.004887 & 0.009072 & 0.093487 & 0.173567 \\
\hline $\mathrm{H} 15$ & 0.018967 & 0.032456 & 0.003964 & 0.006783 & 0.075838 & 0.129772 \\
\hline CPPC (full) & $f_{k}^{+}$ & $f_{k}^{-}$ & $s_{k}^{+}$ & $s_{k}^{-}$ & $\omega_{k}^{+}$ & $\omega_{k}^{-}$ \\
\hline $\mathrm{Cl}$ & 0.037085 & 0.053511 & 0.007751 & 0.011184 & 0.148281 & 0.213958 \\
\hline $\mathrm{C} 2$ & 0.079194 & 0.038498 & 0.016552 & 0.008046 & 0.316649 & 0.15393 \\
\hline C3 & 0.037105 & 0.018265 & 0.007755 & 0.003817 & 0.148361 & 0.073031 \\
\hline $\mathrm{C} 4$ & 0.056432 & 0.033863 & 0.011794 & 0.007077 & 0.225638 & 0.135398 \\
\hline C5 & 0.046093 & 0.054106 & 0.009633 & 0.011308 & 0.184298 & 0.216337 \\
\hline N6 & 0.063725 & 0.047259 & 0.013319 & 0.009877 & 0.254798 & 0.18896 \\
\hline $\mathrm{H} 7$ & 0.05017 & 0.048264 & 0.010486 & 0.010087 & 0.2006 & 0.192979 \\
\hline $\mathrm{H} 8$ & 0.024605 & 0.022564 & 0.005142 & 0.004716 & 0.098381 & 0.09022 \\
\hline H9 & 0.025706 & 0.012418 & 0.005373 & 0.002595 & 0.102783 & 0.049652 \\
\hline H10 & 0.048308 & 0.046734 & 0.010096 & 0.009767 & 0.193155 & 0.186861 \\
\hline C11 & 0.088784 & 0.050459 & 0.018556 & 0.010546 & 0.354994 & 0.201755 \\
\hline $\mathrm{O} 12$ & 0.10292 & 0.103102 & 0.02151 & 0.021548 & 0.411515 & 0.412243 \\
\hline N13 & 0.016223 & 0.049147 & 0.003391 & 0.010272 & 0.064866 & 0.196509 \\
\hline N14 & 0.023381 & 0.043409 & 0.004887 & 0.009072 & 0.093487 & 0.173567 \\
\hline H15 & 0.018967 & 0.032456 & 0.003964 & 0.006783 & 0.075838 & 0.129772 \\
\hline $\mathrm{C} 16$ & 0.07141 & 0.083266 & 0.014925 & 0.017403 & 0.285526 & 0.332931 \\
\hline $\mathrm{C} 17$ & -0.01717 & -0.0153 & -0.00359 & -0.0032 & -0.06865 & -0.06118 \\
\hline $\mathrm{C} 18$ & 0.000227 & -0.00052 & $4.74 E-05$ & -0.00011 & 0.000908 & -0.00206 \\
\hline $\mathrm{C} 19$ & 0.000575 & 0.001021 & 0.00012 & 0.000213 & 0.002299 & 0.004082 \\
\hline C20 & -0.02038 & -0.0215 & -0.00426 & -0.00449 & -0.08147 & -0.08597 \\
\hline $\mathrm{H} 21$ & 0.03456 & 0.04074 & 0.007223 & 0.008515 & 0.138185 & 0.162895 \\
\hline $\mathrm{H} 22$ & 0.039332 & 0.053361 & 0.00822 & 0.011152 & 0.157265 & 0.213359 \\
\hline $\mathrm{H} 23$ & 0.02873 & 0.033782 & 0.006005 & 0.00706 & 0.114874 & 0.135074 \\
\hline $\mathrm{H} 24$ & 0.028555 & 0.033136 & 0.005968 & 0.006925 & 0.114174 & 0.132491 \\
\hline $\mathrm{H} 25$ & 0.029499 & 0.033406 & 0.006165 & 0.006982 & 0.117949 & 0.133571 \\
\hline $\mathrm{H} 26$ & 0.025818 & 0.029323 & 0.005396 & 0.006129 & 0.103231 & 0.117245 \\
\hline $\mathrm{H} 27$ & 0.034231 & 0.046674 & 0.007154 & 0.009755 & 0.136869 & 0.186621 \\
\hline $\mathrm{H} 28$ & 0.025909 & 0.028557 & 0.005415 & 0.005968 & 0.103595 & 0.114182 \\
\hline
\end{tabular}

When a five-membered carbon ring is also attached to isoniazid, it becomes $\mathrm{N}$-cyclopentylidenepyridine4-carbohydrazide. For N-cyclopentylidenepyridine-4carbohydrazide, it has one six-membered pyridine ring (A) and one five-membered carbon ring. The optimized bond length of $\mathrm{C}-\mathrm{C}$ in six-membered pyridine ring $\mathrm{A}$ ranges between $1.405 \AA$ and $1.411 \AA$, while for another ring $\mathrm{B}$ it ranges between $1.521 \AA$ and $1.553 \AA$. The optimized bond lengths of $\mathrm{C}-\mathrm{N}$ in pyridine ring $\mathrm{A}$ range between $1.356 \AA$ and $1.359 \AA$. The optimized bond length of $\mathrm{C}-\mathrm{N}$ in between both rings is found to be $1.392 \AA$. The optimized $\mathrm{C}-\mathrm{H}$ bond lengths attached to the ring $\mathrm{A}$ are found to be $1.084 \AA-1.086 \AA$ while for ring $\mathrm{B}$ it ranges between $1.094 \AA$ and $1.101 \AA$. The optimized $\mathrm{C}=\mathrm{O}$ bond length in between both rings is found to be $1.249 \AA$. The optimized $\mathrm{N}-\mathrm{H}$ bond length in between the rings is found to be $1.017 \AA$. All the optimized bond lengths are in good agreement with the experimental values given in the literature and summarized in Table 1 [7]. This 
TABLE 8: Fukui functions $\left(f_{k}^{+}, f_{k}^{-}\right)$, local softness $\left(s_{k}{ }^{+}, s_{k}{ }^{-}\right)$, and local electrophilicity indices $\left(\omega_{k}{ }^{+}, \omega_{k}^{-}\right)$for selected atomic sites of isoniazid, using Mulliken population analysis at B3LYP/LANL2DZ level.

\begin{tabular}{|c|c|c|c|c|c|c|}
\hline ISO & $f_{k}^{+}$ & $f_{k}^{-}$ & $s_{k}^{+}$ & $s_{k}^{-}$ & $\omega_{k}^{+}$ & $\omega_{k}^{-}$ \\
\hline N6 & 0.087184 & 0.075685 & 0.017594 & 0.015273 & 0.334107 & 0.29004 \\
\hline C11 & 0.045066 & 0.105331 & 0.009094 & 0.021256 & 0.172702 & 0.403649 \\
\hline $\mathrm{O} 12$ & 0.125455 & 0.097778 & 0.025317 & 0.019732 & 0.480769 & 0.374705 \\
\hline N13 & 0.104399 & -0.09059 & 0.021068 & -0.01828 & 0.400078 & -0.34714 \\
\hline H14 & 0.07228 & 0.027223 & 0.014586 & 0.005494 & 0.276991 & 0.104324 \\
\hline H15 & 0.045876 & 0.053631 & 0.009258 & 0.010823 & 0.175806 & 0.205525 \\
\hline H16 & 0.066534 & 0.058946 & 0.013427 & 0.011895 & 0.254972 & 0.225893 \\
\hline N17 & -0.07824 & 0.12441 & -0.01579 & 0.025106 & -0.29982 & 0.476764 \\
\hline ISO (full) & $f_{k}^{+}$ & $f_{k}^{-}$ & $s_{k}^{+}$ & $s_{k}^{-}$ & $\omega_{k}^{+}$ & $\omega_{k}^{-}$ \\
\hline $\mathrm{C} 1$ & 0.070332 & 0.074531 & 0.014193 & 0.01504 & 0.269526 & 0.285618 \\
\hline $\mathrm{C} 2$ & 0.086439 & 0.065308 & 0.017443 & 0.013179 & 0.331252 & 0.250273 \\
\hline $\mathrm{C} 3$ & 0.098962 & -0.00059 & 0.019971 & -0.00012 & 0.379242 & -0.00226 \\
\hline $\mathrm{C} 4$ & 0.068608 & 0.080752 & 0.013845 & 0.016296 & 0.26292 & 0.309458 \\
\hline C5 & 0.061734 & 0.096349 & 0.012458 & 0.019443 & 0.236577 & 0.369229 \\
\hline N6 & 0.087184 & 0.075685 & 0.017594 & 0.015273 & 0.334107 & 0.29004 \\
\hline $\mathrm{H} 7$ & 0.068036 & 0.06207 & 0.01373 & 0.012526 & 0.260728 & 0.237865 \\
\hline $\mathrm{H} 8$ & 0.03037 & 0.040116 & 0.006129 & 0.008095 & 0.116384 & 0.153733 \\
\hline H9 & -0.02115 & 0.070626 & -0.00427 & 0.014252 & -0.08104 & 0.270653 \\
\hline $\mathrm{H} 10$ & 0.068106 & 0.05842 & 0.013744 & 0.011789 & 0.260996 & 0.223877 \\
\hline C11 & 0.045066 & 0.105331 & 0.009094 & 0.021256 & 0.172702 & 0.403649 \\
\hline $\mathrm{O} 12$ & 0.125455 & 0.097778 & 0.025317 & 0.019732 & 0.480769 & 0.374705 \\
\hline N13 & 0.104399 & -0.09059 & 0.021068 & -0.01828 & 0.400078 & -0.34714 \\
\hline H14 & 0.07228 & 0.027223 & 0.014586 & 0.005494 & 0.276991 & 0.104324 \\
\hline H15 & 0.045876 & 0.053631 & 0.009258 & 0.010823 & 0.175806 & 0.205525 \\
\hline H16 & 0.066534 & 0.058946 & 0.013427 & 0.011895 & 0.254972 & 0.225893 \\
\hline N17 & -0.07824 & 0.12441 & -0.01579 & 0.025106 & -0.29982 & 0.476764 \\
\hline
\end{tabular}

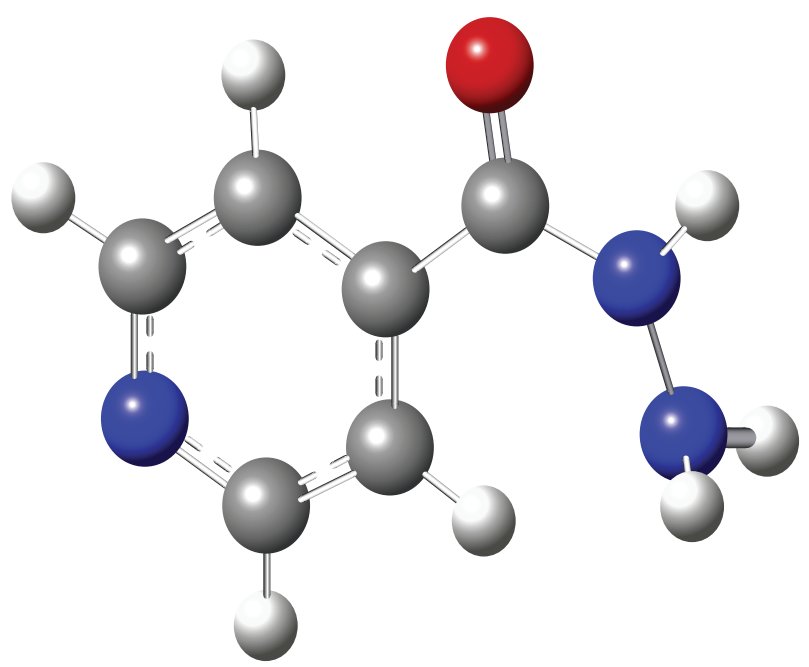

FIGURE 1: Model molecular structure of isoniazid.

difference in the bond lengths is attributed to the difference in bond strength. The length of a chemical bond is the result of equilibrium of attractive and repulsive forces between the atoms which are bonded. In fact, the distance between the atoms of a bond is not constant since the atoms are

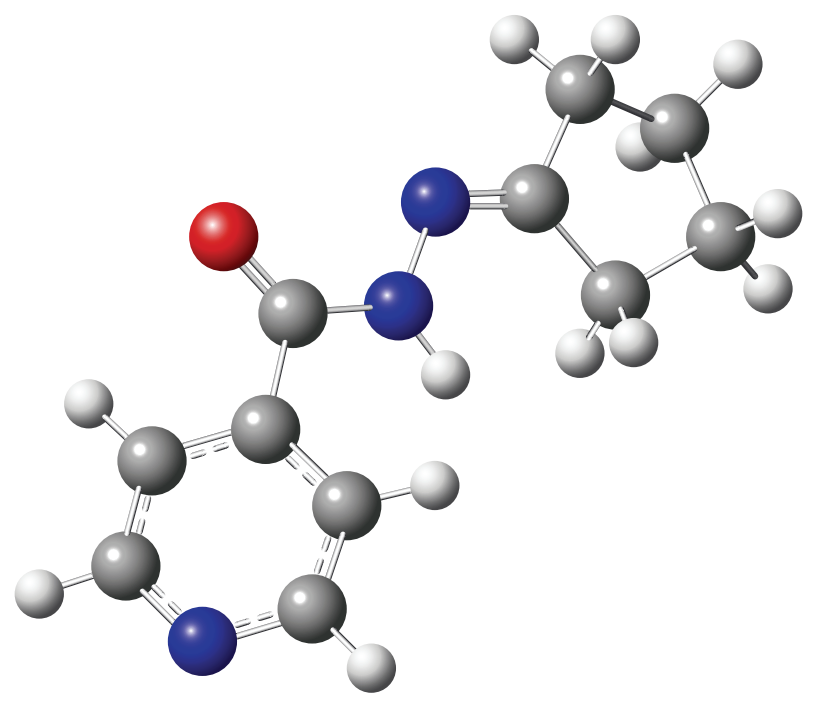

FIGURE 2: Model molecular structure of N-cyclopentylidenepyridine-4-carbohydrazide (CPPC).

always vibrating in molecules; the measured bond distance is, therefore, an average value. Although the measurements may vary in accuracy, still similar bonds have fairly constant 
TABLE 9: Polarizability and hyper polarizability of isoniazid and N-cyclopentylidenepyridine-4-carbohydrazide (CPPC) by (B3LYP)/ LANL2DZ methods.

\begin{tabular}{|c|c|c|c|c|}
\hline & \multicolumn{2}{|c|}{ Isoniazid } & \multicolumn{2}{|c|}{ N-Cyclopentylidenepyridine-4-carbohydrazide } \\
\hline & LANL2DZ & 6-311 G (d, p) & LANL2DZ & $6-311 \mathrm{G}(\mathrm{d}, \mathrm{p})$ \\
\hline \multicolumn{5}{|c|}{ Polarizability } \\
\hline$\alpha x x$ & -54.4243 & -53.1504 & -87.0159 & -86.5468 \\
\hline$\alpha x y$ & -13.9175 & -11.3119 & 8.2530 & 6.5995 \\
\hline$\alpha y y$ & -57.0302 & -56.9567 & -96.2129 & -95.0135 \\
\hline$\alpha y z$ & 0.0007 & 2.9339 & 4.6341 & 4.0900 \\
\hline$\alpha z z$ & -58.4664 & -57.4167 & -88.0328 & -87.9161 \\
\hline$\alpha z x$ & 0.0006 & 2.8602 & 1.0289 & 0.8897 \\
\hline$\alpha$ & 56.6403 & 55.8412 & 90.4205 & 89.8254 \\
\hline \multicolumn{5}{|c|}{ Hyper polarizability } \\
\hline$\beta x x x$ & 104.6160 & 92.6426 & -133.3584 & -99.8131 \\
\hline$\beta x x y$ & -9.7260 & -11.3252 & 50.4571 & 40.4792 \\
\hline$\beta x y y$ & -5.2048 & -2.4771 & -9.9876 & -5.2677 \\
\hline$\beta y y y$ & -28.2262 & -20.4938 & -30.0106 & -21.2944 \\
\hline$\beta z z z$ & 0.0026 & 4.0365 & -0.0107 & -0.6537 \\
\hline$\beta x x z$ & 0.0029 & 8.9620 & 6.7905 & 5.7162 \\
\hline$\beta x z z$ & 13.6068 & 11.5672 & -9.3808 & -4.2219 \\
\hline$\beta y z z$ & -3.0727 & -2.3332 & 1.3678 & 2.3214 \\
\hline$\beta y y z$ & -0.0001 & 2.6541 & 1.3952 & 0.9832 \\
\hline$\beta x y z$ & -0.0020 & -3.8704 & 12.4376 & 10.8998 \\
\hline$\beta$ total & 120.2335 & 115.5787 & 154.1308 & 111.5623 \\
\hline
\end{tabular}

TABLE 10: Calculated thermodynamic properties of isoniazid and N-cyclopentylidenepyridine-4-carbohydrazide (CPPC) by (B3LYP)/ LANL2DZ and 6-311 G (d, p) methods.

\begin{tabular}{|c|c|c|c|c|c|c|}
\hline & \multicolumn{2}{|c|}{$E$ (thermal) $\left(\mathrm{k} \mathrm{cal} \mathrm{mol}^{-1}\right)$} & \multicolumn{2}{|c|}{$\mathrm{CV}\left(\mathrm{cal} \mathrm{K}^{-1} \mathrm{~mol}^{-1}\right)$} & \multicolumn{2}{|c|}{$S\left(\mathrm{cal} \mathrm{K}^{-1} \mathrm{~mol}^{-1}\right)$} \\
\hline & Iso & CPPC & Iso & СРPC & Iso & CPPC \\
\hline \multirow{2}{*}{ Total } & 89.150 & 152.971 & 32.161 & 49.600 & 92.375 & 116.973 \\
\hline & $(88.749)$ & $(151.836)$ & $(32.206)$ & $(50.109)$ & $(92.452)$ & $(117.377)$ \\
\hline \multirow{2}{*}{ Translational } & 0.889 & 0.889 & 2.981 & 2.981 & 40.658 & 41.830 \\
\hline & $(0.889)$ & $(0.889)$ & $(2.981)$ & $(2.981)$ & $(40.658)$ & $(41.830)$ \\
\hline \multirow{2}{*}{ Rotational } & 0.889 & 0.889 & 2.981 & 2.981 & 29.577 & 32.607 \\
\hline & $(0.889)$ & $(0.889)$ & $(2.981)$ & $(2.981)$ & $(29.490)$ & $(32.545)$ \\
\hline \multirow{2}{*}{ Vibrational } & 87.373 & 151.194 & 26.199 & 43.638 & 22.141 & 42.537 \\
\hline & $(86.972)$ & $(150.059)$ & $(26.244)$ & $(44.147)$ & $(22.304)$ & $(43.002)$ \\
\hline
\end{tabular}

lengths in different molecules, variation generally being much less.

\subsection{Vibrational Assignments. The molecules isoniazid and} its derivative (CPPC) contain 17 and 28 atoms and they have 45 and 78 normal modes of vibration, respectively. All the fundamental vibrations are active IR. The harmonic vibrational frequencies are calculated for both the molecules at B3LYP/LANL2DZ and 6-311 G (d, p) level and experimental frequencies (FTIR) have been compared in Tables 2 and 3 for isoniazid and its derivative (CPPC), respectively. Tables 2 and 3 also include the vibrational assignments of the normal modes. Vibrational assignments are based on the observation of the animated modes in GaussView and assignments reported in the literature.
In isoniazid, the $\mathrm{N}-\mathrm{H}$ functional group is present at $3389 \mathrm{~cm}^{-1}$ in calculated spectra which is in good agreement with the experimental data. The $\mathrm{C}-\mathrm{H}$ functional group is present at a number of positions. The stretching vibration, $v(\mathrm{C}-\mathrm{H})$, is expected to occur in the region $2800-3200 \mathrm{~cm}^{-1}$. The $v(\mathrm{C}-\mathrm{H})$ functional group is present at $3079 \mathrm{~cm}^{-1}$ in calculated spectra which is in good agreement with the experimental data, that is, $3050 \mathrm{~cm}^{-1}$. The calculated values of the $v(\mathrm{C}-\mathrm{H})$ vibration lie within this spectral range. The calculated (scaled) and experimental frequencies are deviated by a large amount, which may be explained by the presence of hydrogen bonding in the solid sample. The theoretical calculations have been done on gas-phase molecule.

The $-\mathrm{NH}_{2}$ functional group is important constituents of isoniazid and vibrations corresponding to these groups are 
present in a number of modes. The stretching modes corresponding to these groups are of two types: symmetric and asymmetric stretching. In the symmetric stretching vibration, both the arms of the functional group are stretching in-phase with each other, whereas, in the asymmetric stretching mode, the atomic hydrogen motions are out-of-phase. The twisting and rocking vibrations for both the functional groups are present mixed with other vibrations in middle region. As expected, the ring torsion modes along with wagging modes appear in the low frequency range. An intense band due to torsion of $\mathrm{C}-\mathrm{C}-\mathrm{C}-\mathrm{C}$ is at $658 \mathrm{~cm}^{-1}$ in calculated spectrum which matches well with the experimental one, that is, $560 \mathrm{~cm}^{-1}$. There are some frequencies in lower region having appreciable IR intensity. Furthermore, the study of low frequency vibrations is of great significance, because it gives information on weak intermolecular interactions, which take place in enzyme reactions [14]. Knowledge of low frequency mode is also essential for the interpretation of the effect of electromagnetic radiation on biological systems [15].

In CPPC, the $\mathrm{N}-\mathrm{H}$ functional group is present at $3416 \mathrm{~cm}^{-1}$ in calculated spectra having appreciable IR intensity. The $\mathrm{C}-\mathrm{H}$ functional group is present at a number of positions. The $v(\mathrm{C}-\mathrm{H})$ functional group is present at 2939, 2954 , and $3001 \mathrm{~cm}^{-1}$ in calculated spectra having substantial IR intensity. The region $1600-2000 \mathrm{~cm}^{-1}$ is generally considered as the double bond stretching region for $\mathrm{C}=\mathrm{O}, \mathrm{C}=\mathrm{C}$, and $\mathrm{C}=\mathrm{N}$ bonds [16-19]. The $\mathrm{C}=\mathrm{O}$ stretching vibration, $\nu(\mathrm{C}=\mathrm{O})$, appears as a prominent mode in the calculated spectra at $1604 \mathrm{~cm}^{-1}$.

The $-\mathrm{CH}_{2}$ functional groups are important constituents of CPPC and vibrations corresponding to these groups are present in a number of modes. Due to twist $-\mathrm{CH}_{2}$ mode an intense peak is present at $554 \mathrm{~cm}^{-1}$ in calculated spectra as a prominent mode. The twisting and rocking vibrations for the functional group are present mixed with other vibrations in middle region. Here, we are discussing only most IR active modes present in calculated spectra for both the molecules. The aim of this section is to obtain direct information on lower and higher frequency vibrations of such biologically active molecules. No experimental FTIR spectrum is available for comparison so it will be a suitable path for experimental researchers.

3.3. Electronic Spectra and Electronic Properties. On the basis of fully optimized ground-state structure, TDDFT//B3LYP/LANL2DZ calculations have been used to determine the low-lying excited states of isoniazid and CPPC. The calculated results involving the vertical excitation energies, oscillator strength $(f)$, and wavelength are carried out using the Gaussian 09W program. Experimental wavelengths are not available so these calculated data can help the experimental researchers. Electronic transitions determined from excited state calculations are listed in Table 4 for the three lowest energy transitions of the molecule. For isoniazid, TD-DFT calculation predicts three intense electronic transitions at $5.7714 \mathrm{eV}(214.82 \mathrm{~nm})$, $6.8565 \mathrm{eV}(180.83 \mathrm{~nm})$, and $6.7948 \mathrm{eV}(159.44 \mathrm{~nm})$ with oscillator strengths of $0.1780,0.5155$, and 0.2444 , respectively.

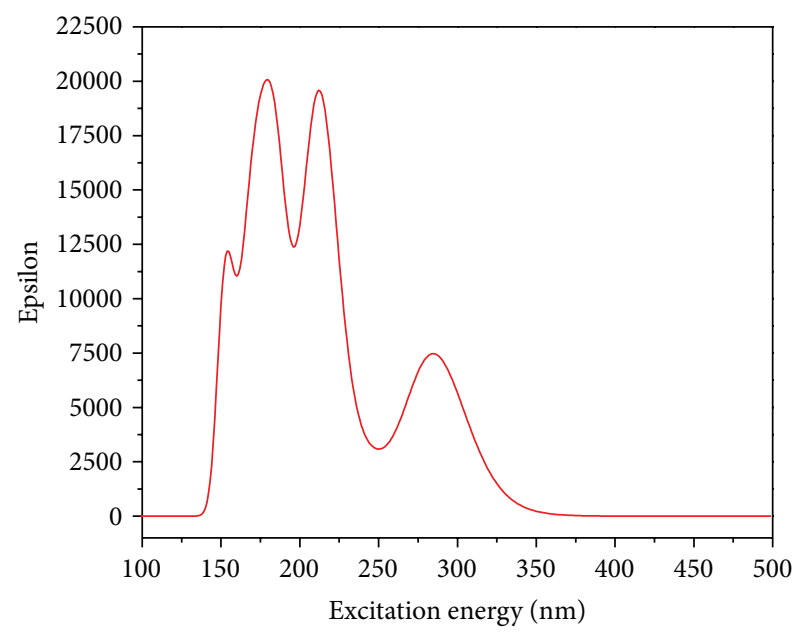

Figure 3: Calculated UV spectrum of CPPC.

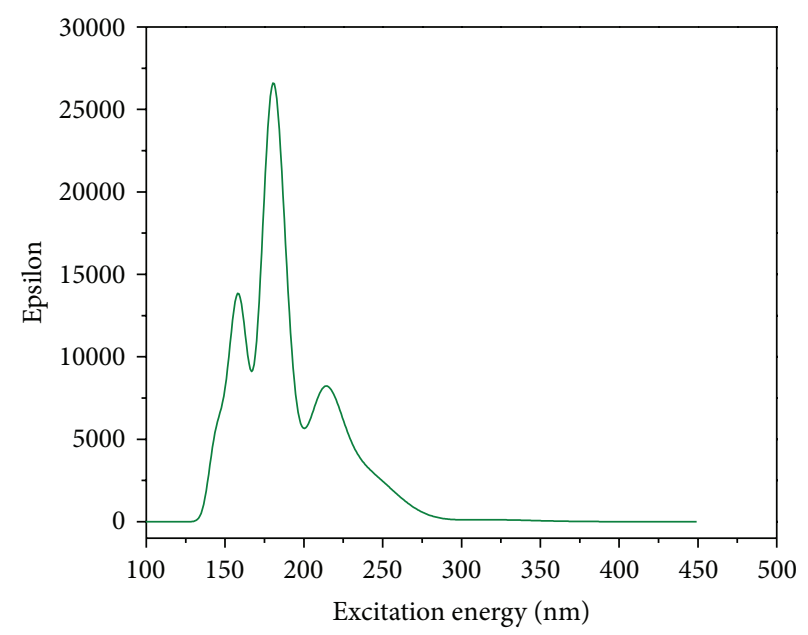

FIGURE 4: Calculated UV spectrum of isoniazid.

For CPPC and TD-DFT calculation predicts three intense electronic transitions at $5.7716 \mathrm{eV}(214.82 \mathrm{~nm}), 6.6955 \mathrm{eV}$ $(185.18 \mathrm{~nm})$, and $7.0662 \mathrm{eV}(175.46 \mathrm{~nm})$ with oscillator strengths of $0.3239,0.2676$, and 0.1706 , respectively. The calculated UV spectrum of isoniazid and its derivative CPPC are shown in Figures 3 and 4.

The frontier orbitals, HOMO and LUMO, determine the way the molecule interacts with other species. The frontier orbital gap helps to characterize the chemical reactivity and kinetic stability of the molecule. A molecule which has a larger orbital gap is more polarized having more reactive part as far as reaction is concerned [20]. According to the present calculations, the frontier orbital gap in case of the given molecules is 4.9531 and $4.7835 \mathrm{eV}$, respectively, for isoniazid and CPPC given in Table 5. So we can say that CPPC is more reactive than isoniazid. The plots of the HOMO, LUMO, and electrostatic potential for both the molecules are shown in Figures 5-10. For isoniazid, HOMO (Figure 5) is located over the whole molecule. LUMO is located (Figure 6) at the same place as HOMO excluding hydrogen atoms. For 


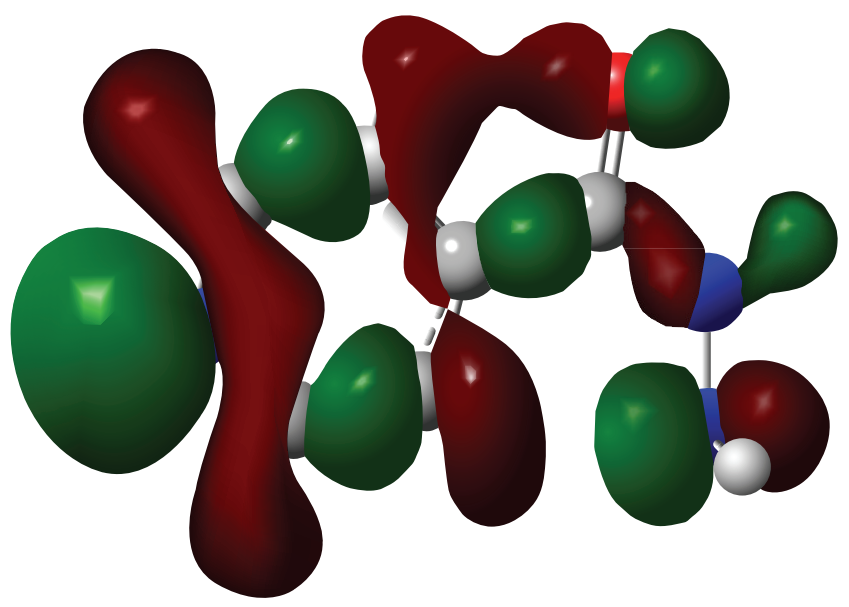

FIgURE 5: HOMO picture of isoniazid.

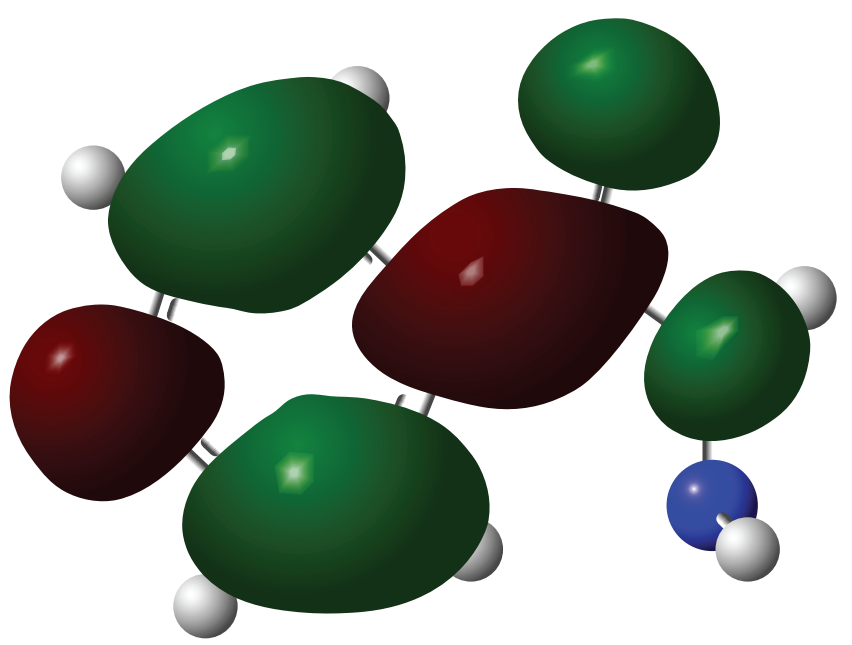

FIgURE 6: LUMO picture of isoniazid.

CPPC, HOMO (Figure 7) is located nearby and on ring B. LUMO is located (Figure 8) nearby and on ring A. The importance of MESP lies in the fact that it simultaneously displays molecular size, shape, and positive, negative, and neutral electrostatic potential regions in terms of colour grading (shown in Figures 9 and 10) and is very useful in investigation of molecular structure with its physiochemical property relationship [21-25]. We have also plotted 3D MESP structures of the title compound as shown in Figures 11 and 12. For isoniazid and CPPC, the electronegative region is outside of the molecule near the oxygen atoms. The energy equal to the shielded potential energy surface is required for any substitution reaction near oxygen. The electronegative lines (in between -0.08 a.u. and -0.02 a.u.) form a closed contour which clearly indicates that total flux passing in between these curves are not equal to zero. For any nucleophilic substitution reaction near oxygen (closed contour area), an amount of energy equal to shielded potential energy surface is required; however, the remaining part of the molecule is suitable for electrophilic substitution reaction. Thus, it can be asserted that MESP values have been shown to be well related to biological properties. [26-28].

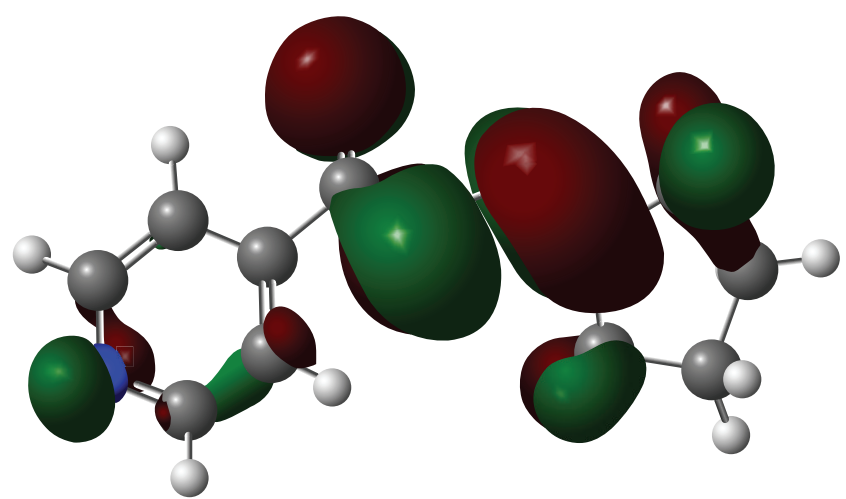

Figure 7: HOMO picture of N-cyclopentylidenepyridine-4carbohydrazide (CPPC).

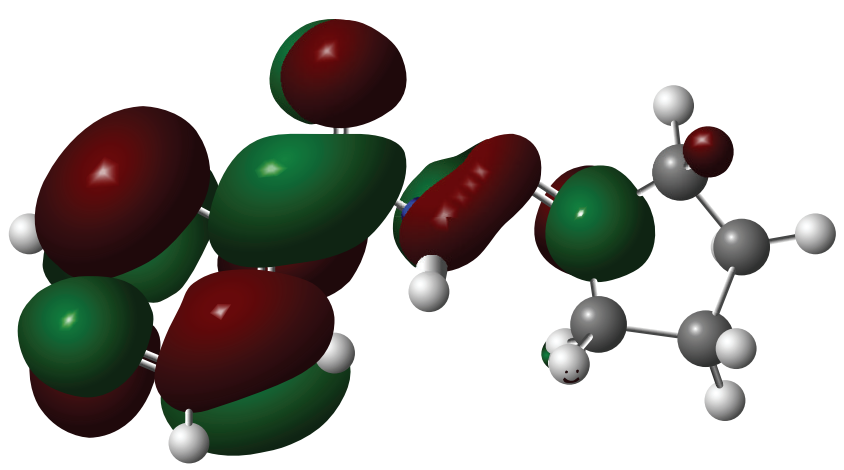

FIGURE 8: LUMO picture of N-cyclopentylidenepyridine-4carbohydrazide (CPPC).

\subsection{Reactivity Descriptors}

3.4.1. Global Reactivity Descriptors. The energies of frontier molecular orbitals ( $\left.{ }^{\varepsilon} \mathrm{HOMO},{ }^{\varepsilon} \mathrm{LUMO}\right)$, energy band gap $\left({ }^{\varepsilon} \mathrm{LUMO}-{ }^{\varepsilon} \mathrm{HOMO}\right)$, electronegativity $\left(\chi=-(1 / 2)\left({ }^{\varepsilon} \mathrm{LUMO}+{ }^{\varepsilon} \mathrm{HOMO}\right)\right)$, chemical potential $(\mu=-\chi)$, global hardness $\left(\eta=(1 / 2)\left({ }^{\varepsilon} \mathrm{LUMO}-{ }^{\varepsilon} \mathrm{HOMO}\right)\right)$, global softness $(S=1 / 2 \eta)$, and global electrophilicity index $\left(\omega=\mu^{2} / 2 \eta\right)$ [29-32] of isoniazid and CPPC have been listed in Table 6. It is found that the gap between HOMO and LUMO is smaller in CPPC than isoniazid.

3.4.2. Local Reactivity Descriptors. Fukui function (FF) of a molecule provides information of the local site reactivity within the molecule and as such it provides a method for understanding and categorizing chemical reactions. These values represent the qualitative descriptors of reactivity of different atoms in the molecule. Ayers and Parr [33] have elucidated that molecules tend to react where the FF is the largest when attacked by soft reagents and in places where the FF is smaller when attacked by hard reagents. The use of Fukui functions for the site selectivity of isoniazid and CPPC molecules for nucleophilic and electrophilic attacks has been made with special emphasis on the dependence of Fukui values on the basis of B3LYP/6-311G(d,p) level of theory. Using Mulliken atomic charges of cationic and anionic states 


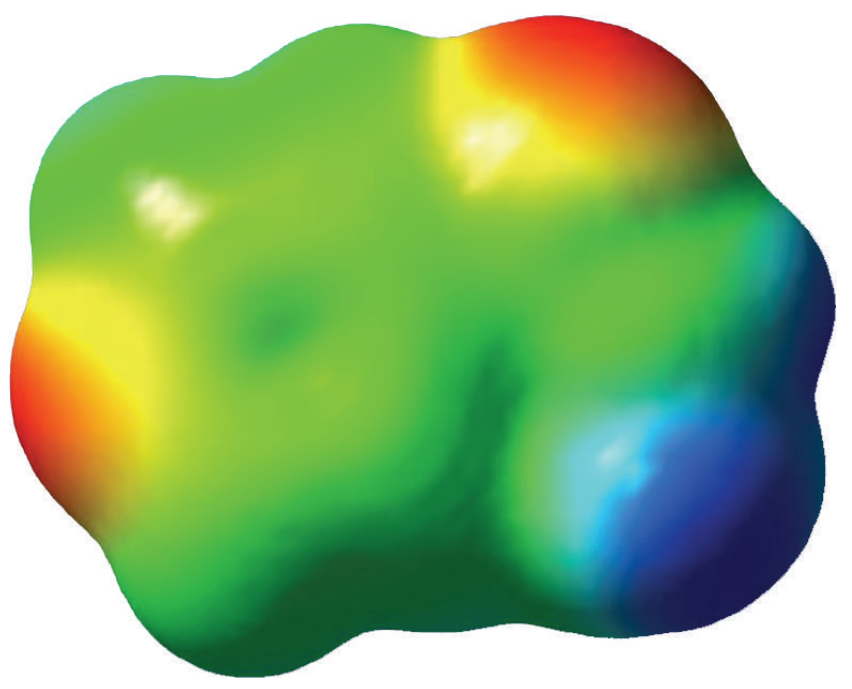

FIGURE 9: MESP picture of isoniazid.

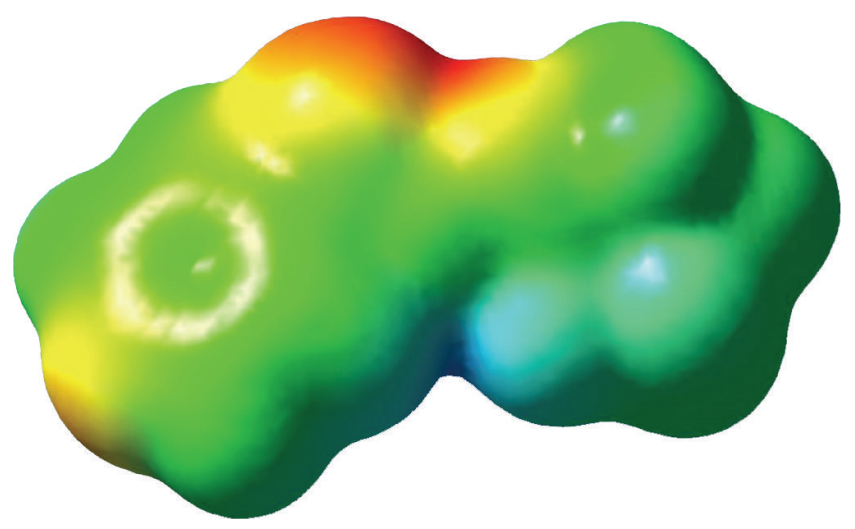

FIgURE 10: MESP picture of N-cyclopentylidenepyridine-4carbohydrazide (CPPC).

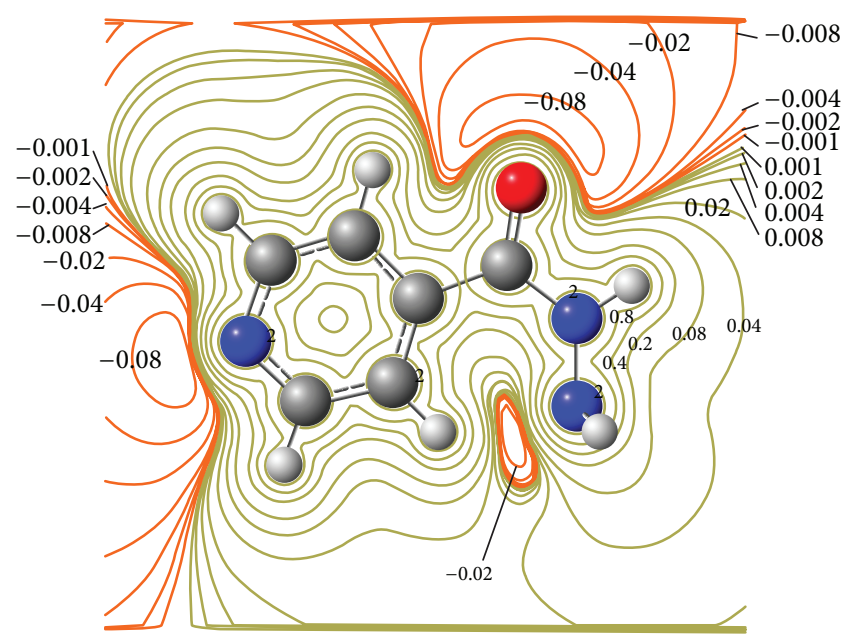

FIGURE 11: Contour plot of isoniazid.

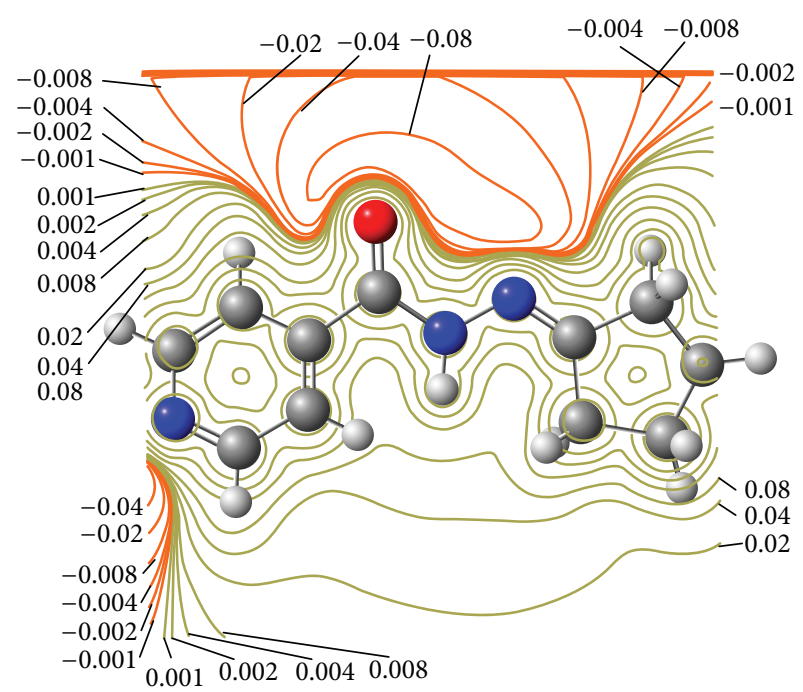

FIgURE 12: Contour plot of N-cyclopentylidenepyridine-4carbohydrazide (CPPC).

of isoniazid and CPPC, local Fukui functions $\left(f k^{+}, f k^{-}\right)$, local softness $\left(s k^{+}, s k^{-}\right)$, and local electrophilicity indices $\left(\omega k^{+}, \omega k^{-}\right)[30,31]$ have been calculated using the following equation:

$$
\begin{aligned}
& f k^{+}=[q(N+1)-q(N)] \quad \text { for nucleophilic attack, } \\
& f k^{-}=[q(N)-q(N-1)] \quad \text { for electrophilic attack, } \\
& f k^{0}=\frac{1}{2}[q(N+1)+q(N-1)] \quad \text { for radical attack. }
\end{aligned}
$$

Local softness and electrophilicity indices are calculated using the following equation:

$$
\begin{array}{ll}
s k^{+}=S f k^{+}, & s k^{-}=S f k^{-}, \\
\omega k^{+}=\omega f k^{+}, & \omega k^{-}=\omega f k^{-},
\end{array}
$$

where + and - signs show nucleophilic and electrophilic attacks, respectively.

Fukui functions, local softness, and local electrophilicity indices for selected atomic sites in isoniazid and CPPC have been listed in Tables 7 and 8. For CPPC, the maximum values for local nucleophilic reactivity descriptors $\left(f k^{-}, s k^{-}\right.$, $\omega k^{-}$) at N13 and N14 indicate that these two sites are more prone to electrophilic attack. But on comparison with N17 of isoniazid it is found that the N14 of CPPC has lower affinity towards electrophile. This situation changes because the nitrogen of CPPC is bonded through a double bond to the $\mathrm{C} 16$ of a pentagonal ring; hence, the charge density around N14 decreases.

3.5. Dipole Moment, Polarizability, Hyper Polarizability, and Thermodynamic Properties. Dipole moment $(\mu)$, polarizability $\langle\alpha\rangle$, and total first static hyper polarizability $\beta[31,32]$ are also calculated in Tables 5 and 9 by using density functional 
theory. They can be expressed in terms of $x, y, z$ components and are given by the following equation:

$$
\begin{gathered}
\mu=\left(\mu_{x}^{2}+\mu_{y}^{2}+\mu_{z}^{2}\right)^{1 / 2} \\
\langle\alpha\rangle=\frac{1}{3}\left[\alpha_{x x}+\alpha_{y y}+\alpha_{z z}\right] \\
\beta_{\text {Total }}=\left(\beta_{x}^{2}+\beta_{y}^{2}+\beta_{z}^{2}\right)^{1 / 2} \\
=\left[\left(\beta_{x x x}+\beta_{x y y}+\beta_{x z z}\right)^{2}+\left(\beta_{y y y}+\beta_{y x x}+\beta_{y z z}\right)^{2}\right. \\
\left.+\left(\beta_{z z z}+\beta_{z x x}+\beta_{z y y}\right)^{2}\right]^{1 / 2}
\end{gathered}
$$

The $\beta$ components of Gaussian output are reported in atomic units, where 1 a.u. $=8.3693 \times 10^{-33}$ e.s.u. For isoniazid and CPPC, the calculated dipole moment values are 5.49 and 6.25 Debye. Having higher dipole moment than water (2.16 Debye), isoniazid and CPPC both can be used as better solvents. For isoniazid, we see a greater contribution of $\alpha_{z z}$ in molecule which shows that molecule is elongated more towards $Z$ direction and more contracted to $X$ direction. Perpendicular part contributes less part of polarizability of molecule. $\mathrm{B}_{x x x}, \beta_{y y y}$ contribute a larger part in hyper polarizability. This shows that molecule is more optical active in $X$-axis and $Y$-axis directions. For CPPC, we see a greater contribution of $\alpha_{y y}$ in molecule which shows that molecule is elongated more towards $Y$ direction and more contracted to $X$ direction. Perpendicular part contributes less part of polarizability of molecule.

$\mathrm{B}_{x x x}, \beta_{x x y}$ contribute a larger part in hyper polarizability. This shows that molecule is more optical active in $X$-axis and $X Y$-plane directions. The connection between the electric dipole moments of an organic molecule having donoracceptor substituent and first order hyper polarizability is widely recognized in the literature. Standard thermodynamic functions such as free energy, constant volume heat capacity $C V$, and entropy $S$ have also been calculated for both the molecules and are given in Table 10. These functions can provide helpful information for further study of the title compounds.

\section{Conclusion}

This paper reports a comprehensive computational structural study on antituberculosis drug isoniazid and its new derivative N-cyclopentylidenepyridine-4-carbohydrazide. The frequency assignments for isoniazid and its derivative $\mathrm{N}$ cyclopentylidenepyridine-4-carbohydrazide have been done for the first time by employing density functional theory (DFT) with LANL2DZ and 6-311 G (d, p) as the basis sets. Normal modes analysis provides detailed description of the vibrational spectra of the molecules in question. Reactivity reflects the susceptibility of a substance towards a specific chemical reaction and plays a key role in, for example, the design of new molecules and understanding biological systems and material science. The lower value of frontier orbital energy gap and a higher dipole moment in case of CPPC suggest a more reactive nature as compared to isoniazid. Hyper polarizability is mainly controlled by the planarity of the molecules, the donor and accepter strength, and bond length alteration. The values of hyper polarizability indicate a possible use of these compounds in electrooptical applications.

We have also discussed global and local reactivity descriptors sites for both molecules during electrophilic, nucleophilic, and radical attacks. These values represent the qualitative descriptors of reactivity of different atoms in the molecule. The present work might encourage the need for an extensive study by the experimentalists interested in the vibrational spectra and the structure of these compounds. The results reported in the present paper can help in the experimental investigations on the origin of the biological activity of these molecules.

\section{Conflict of Interests}

The authors declare that there is no conflict of interests regarding the publication of this paper.

\section{References}

[1] H. Meyer and J. Mally, "On hydrazine derivatives of pyridine carbonic acids," Monatshefte für Chemie und Verwandte Teile Anderer Wissenschaften, vol. 33, pp. 393-414, 1912.

[2] H. L. Rieder, "Fourth-generation fluoroquinolones in tuberculosis," The Lancet, vol. 373, no. 9670, pp. 1148-1149, 2009.

[3] S. Shimizu, N. Watanabe, T. Kataoka et al., "Pyridine and pyridine derivatives," in Ullmann's Encyc Lopedia of Industrial Chemistry, John Wiley \& Sons, New York, NY, USA, 2007.

[4] P. B. Issopolus and P. T. Economou, "A sensitive colorimetric determination of microquantities of isonicotinic acid hydrazide (isoniazid)," International Journal of Pharmaceutics, vol. 57, pp. 235-239, 1989.

[5] S. Broussy, V. Bernardes-Génisson, Y. Coppel, A. Quémard, J. Bernadou, and B. Meunier, "1H and 13C NMR characterization of pyridinium-type isoniazid-NAD adducts as possible inhibitors of InhA reductase of Mycobacterium tuberculosis," Organic and Biomolecular Chemistry, vol. 3, no. 4, pp. 670-673, 2005.

[6] S. Gunasekaran, E. Sailatha, S. Seshadri, and S. Kumaresan, "FTIR, FT Raman spectra and molecular structural confirmation of isoniazid," Indian Journal of Pure and Applied Physics, vol. 47, no. 1, pp. 12-18, 2009.

[7] A. Lemmerer, J. Bernstein, and V. Kahlenberg, " $\mathrm{N}$ ' cyclopentylidenepyridine-4-carbohydrazide," Acta Crystallographica E, vol. 68, article 01299, 2012.

[8] P. Hohenberg and W. Kohn, "Inhomogeneous electron gas," Physical Review, vol. 136, no. 3, pp. B864-B871, 1964.

[9] A. D. Becke, "Density-functional thermochemistry. III. The role of exact exchange," The Journal of Chemical Physics, vol. 98, no. 7, pp. 5648-5652, 1993.

[10] C. Lee, W. Yang, and R. G. Parr, "Development of the ColleSalvetti correlation-energy formula into a functional of the electron density," Physical Review B, vol. 37, no. 2, pp. 785-789, 1988. 
[11] M. J. Frisch, G. W. Trucks, H. B. Schlegel et al., Gaussian 09, Gaussian, Pittsburgh, Pa, USA, 2009.

[12] P. L. Fast, J. C. Corchado, M. L. Sánchez, and D. G. Truhlar, "Optimized parameters for scaling correlation energy," Journal of Physical Chemistry A, vol. 103, no. 17, pp. 3139-3143, 1999.

[13] A. Frisch, A. B. Nelson, and A. J. Holder, Gauss View, Gauss, Pittsburgh, Pa, USA, 2000.

[14] K.-C. Chou, "Biological functions of low-frequency vibrations (phonons). III. Helical structures and microenvironment," Biophysical Journal, vol. 45, no. 5, pp. 881-889, 1984.

[15] H. Frohlich, Biological Coherence and Response to External Stimuli, Springer, Berlin, Germany, 1988.

[16] M. Alcolea Palafox, G. Tardajos, A. Guerrero-Martínez et al., "FT-IR, FT-Raman spectra, density functional computations of the vibrational spectra and molecular geometry of biomolecule 5-aminouracil," Chemical Physics, vol. 340, no. 1-3, pp. 17-31, 2007.

[17] J. S. Singh, "FTIR and Raman spectra and fundamental frequencies of biomolecule: 5-Methyluracil (thymine)," Journal of Molecular Structure, vol. 876, no. 1-3, pp. 127-133, 2008.

[18] C. P. Beetz Jr. and G. Ascarelli, "The low frequency vibrations of pyrimidine and purine bases," Spectrochimica Acta A, vol. 36, no. 3, pp. 299-313, 1980.

[19] J. Bandekar and G. Zundel, "The role of CO transition dipoledipole coupling interaction in uracil," Spectrochimica Acta A, vol. 39, no. 4, pp. 337-341, 1983.

[20] I. Fleming, Frontier Orbitals and Organic Chemical Reactions, John Wiley \& Sons, New York, NY, USA, 1976.

[21] J. S. Murray and K. Sen, Molecular Electrostatic Potentials, Concepts and Applications, Elsevier, Amsterdam, The Netherlands, 1996.

[22] I. Alkorta and J. J. Perez, "Molecular polarization potential maps of the nucleic acid bases," International Journal of Quantum Chemistry, vol. 57, no. 1, pp. 123-135, 1996.

[23] E. Scrocco and J. Tomasi, in Advances in Quantum Chemistry, P. Lowdin, Ed., Academic Press, New York, NY, USA, 1978.

[24] F. J. Luque, M. Orozco, P. K. Bhadane, and S. R. Gadre, "SCRF calculation of the effect of hydration on the topology of the molecular electrostatic potential," Journal of Physical Chemistry, vol. 97, no. 37, pp. 9380-9384, 1993.

[25] J. Šponer and P. Hobza, "DNA base amino groups and their role in molecular interactions: ab initio and preliminary density functional theory calculations," International Journal of Quantum Chemistry, vol. 57, no. 5, pp. 959-970, 1996.

[26] M. Bohl, K. Ponsold, and G. Reck, "Quantitative structureactivity relationships of cardiotonic steroids using empirical molecular electrostatic potentials and semiempirical molecular orbital calculations," Journal of Steroid Biochemistry, vol. 21, no. 4, pp. 373-379, 1984.

[27] D. F. lewis and V. Griffiths, "Xenobiotica, the fate of foreign compounds in biological systems," Informa Healthcare, vol. 17, pp. 769-776, 1987.

[28] A. Kumar and P. C. Mishra, "Structure-activity relationships for some anti-HIV drugs using electric field mapping," Journal of Molecular Structure, vol. 277, pp. 299-312, 1992.

[29] L. A. Flippin, D. W. Gallagher, and K. Jalali-Araghi, "A convenient method for the reduction of ozonides to alcohols with borane-dimethyl sulfide complex," Journal of Organic Chemistry, vol. 54, no. 6, pp. 1430-1432, 1989.

[30] R. G. Parr, L. V. Szentpály, and S. Liu, "Electrophilicity index," Journal of the American Chemical Society, vol. 121, no. 9, pp. 1922-1924, 1999.
[31] P. K. Chattaraj and S. Giri, "Stability, reactivity, and aromaticity of compounds of a multivalent superatom," Journal of Physical Chemistry A, vol. 111, no. 43, pp. 11116-11121, 2007.

[32] J. Padmanabhan, R. Parthasarathi, V. Subramanian, and P. K. Chattaraj, "Electrophilicity-based charge transfer descriptor," Journal of Physical Chemistry A, vol. 111, no. 7, pp. 1358-1361, 2007.

[33] P. W. Ayers and R. G. Parr, "Variational principles for describing chemical reactions: the Fukui function and chemical hardness revisited," Journal of the American Chemical Society, vol. 122, no. 9, pp. 2010-2018, 2000. 

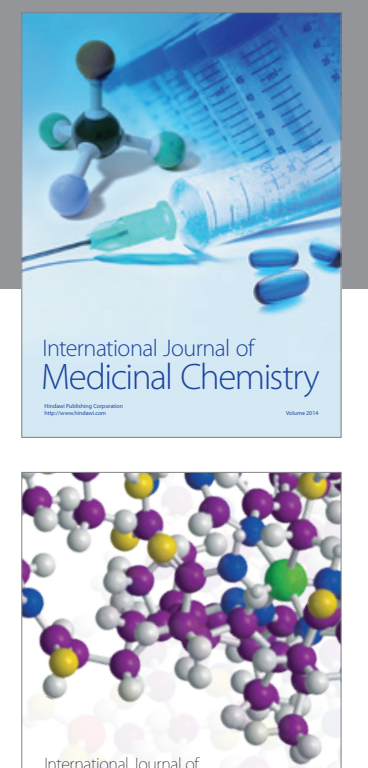

\section{Carbohydrate} Chemistry

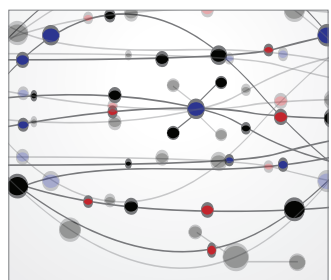

The Scientific World Journal
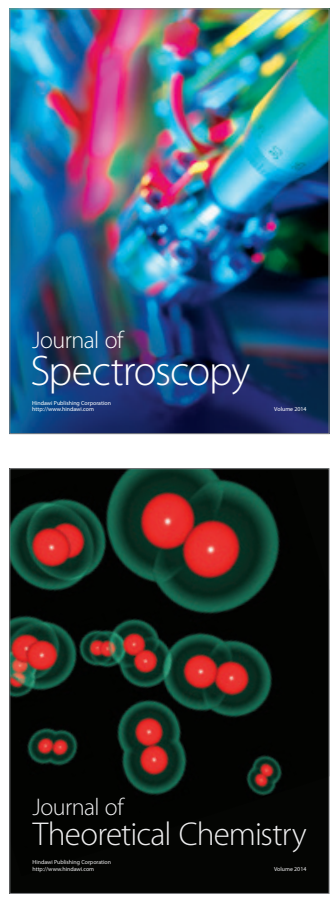
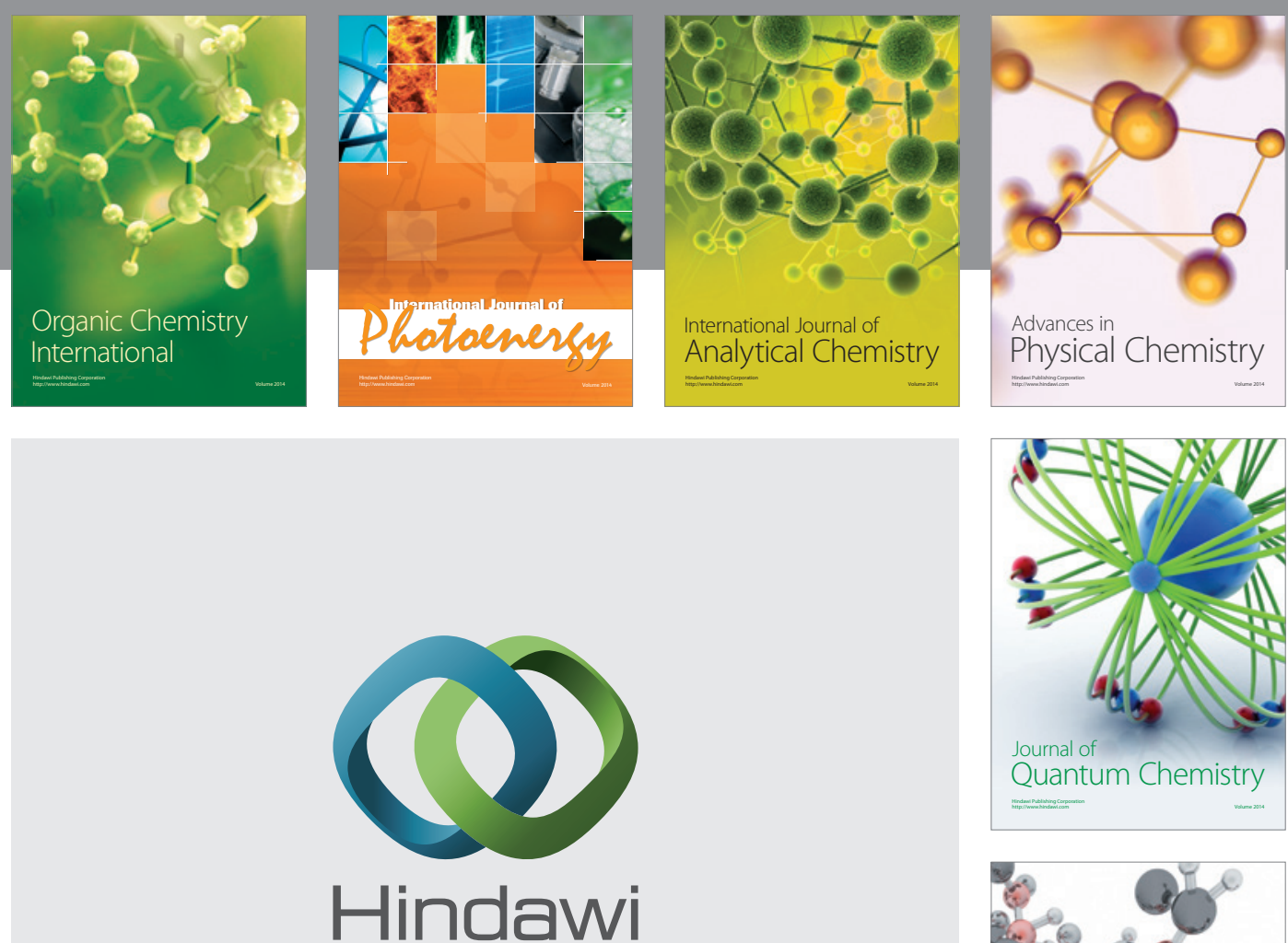

Submit your manuscripts at

http://www.hindawi.com

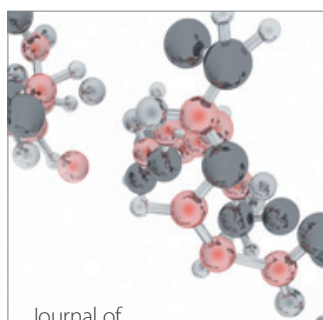

Analytical Methods

in Chemistry

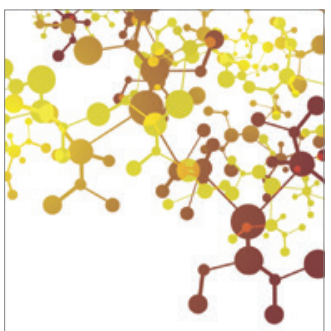

Journal of

Applied Chemistry

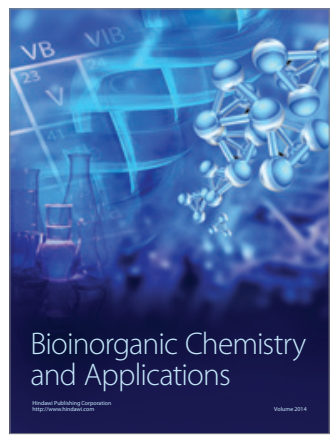

Inorganic Chemistry
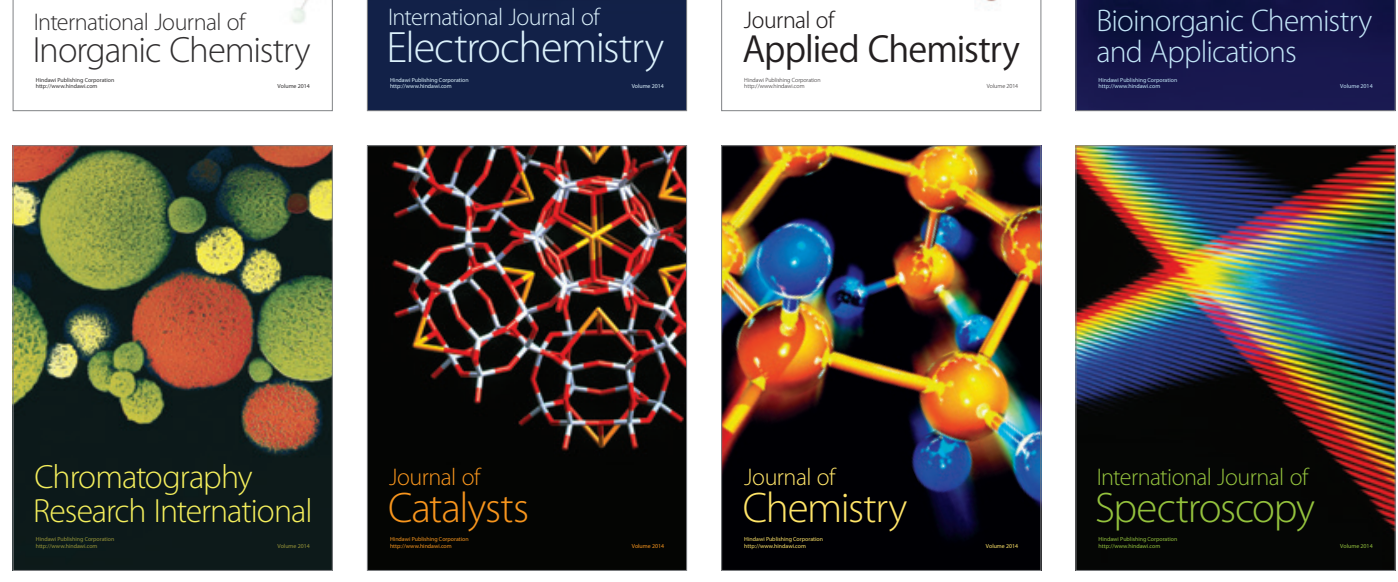